\title{
Cancer screening behaviours among South Asian immigrants in the UK, US and Canada: a scoping study
}

\author{
Crawford J, Ahmad F, Beaton D \& Bierman AS
}

"This is the peer reviewed version of the following article:

Crawford J, Ahmad F, Beaton D \& Bierman AS. Cancer screening behaviours among South Asian immigrants in the UK, US, and Canada: a scoping study. Journal of Health and Social Care in the Community 2015; 24:123-153.

has been published in final form at:

DOI: $10.1111 / h s c .12208$.

This article may be used for non-commercial purposes in accordance with Wiley Terms and Conditions for Self-Archiving." 


\begin{abstract}
South Asian (SA) immigrants settled in the United Kingdom (UK) and North America [United States (US) and Canada] have low screening rates for breast, cervical and colorectal cancers. Incidence rates of these cancers increase among SA immigrants after migration, becoming similar to rates in non-Asian native populations. However, there are disparities in cancer screening, with low cancer screening uptake in this population. We conducted a scoping study using Arksey \& O'Malley's framework to examine cancer screening literature on SA immigrants residing in the UK, US and Canada. Eight electronic databases, key journals and reference lists were searched for English language studies and reports. Of 1465 identified references, 70 studies from 1994 to November 2014 were included: $63 \%$ on breast or cervical cancer screening or both; $10 \%$ examined colorectal cancer screening only; $16 \%$ explored health promotion/service provision; $8 \%$ studied breast, cervical and colorectal cancer screening; and 3\% examined breast and colorectal cancer screening. A thematic analysis uncovered four dominant themes: (i) beliefs and attitudes towards cancer and screening included centrality of family, holistic healthcare, fatalism, screening as unnecessary and emotion-laden perceptions; (ii) lack of knowledge of cancer and screening related to not having heard about cancer and its causes, or lack of awareness of screening, its rationale and/or how to access services; (iii) barriers to access including individual and structural barriers; and (iv) gender differences in screening uptake and their associated factors. Findings offer insights that can be used to develop culturally sensitive interventions to minimise barriers and increase cancer screening uptake in these communities, while recognising the diversity within the SA culture. Further research is required to address the gap in colorectal cancer screening literature to more fully understand SA immigrants' perspectives, as well as research to better understand gender-specific factors that influence screening uptake.
\end{abstract}

Key words: access to healthcare, cancer prevention and control, ethnic minorities, ethnicity and health, screening, South Asian 


\section{Bullets Section}

What is known about this topic?

- Over time, South Asian immigrants who settle in western countries have similar rates of cancer incidence for breast, cervical and colorectal cancers as native-born populations. Population-based breast, cervical and colorectal cancer screening is recommended for early detection. Yet, disparities in screening uptake among South Asian immigrants persist.

- An understanding of the sociocultural context influencing cancer screening uptake is needed to develop effective programmes to improve cancer screening rates among South Asian immigrants.

\section{What this paper adds?}

- An examination of the sociocultural context of South Asian immigrants' beliefs and attitudes towards cancer screening elucidated the need to consider family and holistic beliefs in the development of health-promoting messages; to increase knowledge about risk factors and cancer screening benefits; and to address health system barriers to increase screening uptake.

- Public health and cancer care practitioners should involve South Asian immigrants in the development of community-based programming to address local needs with the aim of increasing screening uptake.

- There is limited evidence about factors influencing uptake of (or participation in) colorectal cancer screening including gender-specific factors among South Asian immigrants. 


\section{Introduction}

Population-based cancer screening for breast, cervical and colorectal cancer has the potential to reduce mortality and morbidity if performed as per guideline recommendations in the general average risk population (United States Preventive Services Task Force 2008, 2009 , 2012). However, rates of uptake for breast, cervical or colorectal cancers among ethnic minority populations in the United Kingdom (UK), United States (US) and Canada are sub-optimal (Quan et al. 2006, Szczepura et al. 2008, Lee et al. 2010a). South Asian (SA) immigrants form a growing community in the UK, US and Canada (Statistics Canada 2008, US Census Bureau 2010, UK Census 2011). SA immigrants also represent a diverse community with ancestral origins largely from the Indian subcontinent including India, Bangladesh, Pakistan, Sri Lanka and smaller numbers from the diaspora, originating from countries such as South or East Africa and the Caribbean (Ballard 2003, Tran et al. 2005). The incidence rates of breast and colorectal cancers among SA immigrants residing in the UK and North America are comparable to those in non-Asian-born populations (Smith et al. 2003, Jain et al. 2005, Hislop et al. 2007, Hossain et al. 2008, Rastogi et al. 2008; Virk et al. 2010). Yet, disparities in cancer screening have been documented with SA immigrants having low rates of breast, cervical and colorectal cancer screening (Szczepura et al. 2008, Lee et al. 2010a, Lofters et al. 2010). Thus, SA immigrants are at risk for avoidable morbidity and mortality from these cancers.

While prior reviews have examined cancer screening-related barriers among ethnic minority populations in the UK, US and Canada (Wu et al. 2005, Elkan et al. 2006, Johnson et al. 2008, Hanson et al. 2009, Alexandraki \& Mooradian 2010, Sokal 2010), they have focused on studies in one country, one or two population-based cancer screening modalities (i.e. breast, cervical) or excluded SA immigrants. Barriers to cancer screening among SA immigrants include individual and structural barriers. Individual barriers to cancer screening or access to health services reflect issues not always under the control of the individual (Baron et al. 2008). The individual barriers to screening encountered by SA immigrants include lack of knowledge and access, low self-perceived risk, loss of social networks, language barriers and competing priorities of work and family (Ahmad et al. 2004, Oelke \& Vollman 2007). Structural barriers include health policy, socioeconomic factors, health insurance coverage and systemic health service provision, such as usual source of care (family physician), screening service hours of operation, local access to services or transportation (Baron et al. 2008). SA immigrants identified structural barriers to cancer screening such as lack of local access (Thomas et al. 2005) and lack of physician recommendation (Somanchi et al. 2010).

To address health inequities related to low cancer screening among SA immigrants, an understanding of the sociocultural context including beliefs and attitudes, and facilitators and barriers to cancer screening in these populations is required. To this end, a scoping study utilising Arksey \& O'Malley's (2005) framework was undertaken. This framework provides a structured method to develop a comprehensive understanding of current knowledge, and to identify knowledge gaps through the examination of diverse and heterogeneous literature. In this scoping study, the research question was: What are the cancer screening beliefs, attitudes and behaviours of SA immigrants residing in the UK, US and Canada? The intended outcome was a synthesis of existing knowledge about barriers and facilitators to cancer screening in these populations to inform current practice, policy and future research.

\section{Methods}

Arksey \& O'Malley's (2005) framework encompasses five stages: (i) research question formulation; (ii) a comprehensive literature search and development of relevancy criteria; (iii) identification of relevant stud- 
ies; (iv) charting of extracted data from included studies and reports; and (v) summarising and reporting of findings. This method is advantageous as it incorporates not only a transparent and reproducible search strategy but also enables an examination of a broad research question by the inclusion of a variety of study designs and development of study selection criterion in an iterative manner. A narrative review that employed thematic analysis was the approach used as a process to produce a simplified synthesis of included studies or reports (Mays et al. 2005). The narrative review process aims to present findings as they are reported in the literature and does not aim to transform data. This method is comprehensive, flexible and efficient because it allows different types of evidence to be used to identify main concepts related to a specific research topic that has not undergone prior review. To synthesise evidence, a thematic analysis of dominant recurring and important themes from the literature was undertaken to address the research question (Mays et al. 2005, Levac et al. 2010). The narrative review method utilising thematic analysis gives high importance to the relevance of literature and does not attempt to assess the quality of studies.

For this study, the concept of population-based cancer screening included breast, cervical and colorectal cancers. The health outcome of interest, cancer screening behaviours, encompassed: beliefs and attitudes towards cancer and screening; barriers and facilitators to cancer prevention; reasons for screening; and cancer screening uptake. The target population was SA immigrants defined as individuals who originate from the southern part of Asia or who claim a cultural ancestry or origin from India, Pakistan, Bangladesh, Sri Lanka or Nepal, and may include ethnic backgrounds from diverse ancestries, such as Hindu, Goan, Gujarati, Nepali, Sikh, Punjabi, Pakistani or Tamil (Tran et al. 2005).

A librarian was consulted for the literature review process and refinement of the search strategy, and a primary reviewer became familiar with the literature. An interdisciplinary team comprised of a public health practitioner with oncology certification and experience working with immigrant populations; a medical health professional and health services researcher; a public health researcher with a focus on immigrant communities including SAs; and an occupational therapist and clinical epidemiologist with interest in disease prevention and cross-cultural adaptation of materials and measures. All members were involved in decisions surrounding inclusion and exclusion criteria, and refinement of themes.
A literature search was initially conducted in June 2012 and was updated in November 2014. English language studies and reports were searched using the following electronic databases: Ovid MEDLINE [1946-October Week 5 2014], EMBASE [1980-2014 Week 45], PsychoINFO [1806-November Week 1 2014], CINHAL, PubMed, the Cochrane Library [Issue 11 of 12, November 2014], Scopus and System for Information on Grey Literature in Europe. Six key journal volumes and issues were searched electronically from January 2005 to November 2014 inclusively: Asian Pacific Journal of Cancer Prevention; Canadian Journal of Public Health; Cancer; Journal of Immigrant and Minority Health; Journal of Medical Screening; and Social Science and Medicine. The Web of Science was also searched using relevant studies included in the scoping study because of the potential to yield further citations (Ahmad et al. 2005, De Alba et al. 2005, Asanin and Wilson 2007, Robb et al. 2008, Szczepura et al. 2008, Glenn et al. 2009, Taskila et al. 2009, Pourat et al. 2010). Reference lists of included studies were also searched, as well as key websites of evidence-based reports, for example, Cancer Care Ontario, Public Health Agency of Canada, Ontario Women's Health Evidence-Based Report, the Council of Agencies Servicing SA in Ontario and the UK Bowel Screening Programme.

The main subject headings and key word search terms used were as follows: 'Asian', 'Asian Continental Ancestry Group', 'Asian American or British Asian or Indian', 'Hindu', 'Bangladesh', 'Sri Lanka', 'emigrants and immigrant', 'illegal immigrant', 'migrant', 'refugee', 'cancer screening', 'mass screening', 'cancer prevention', 'early detection of cancer' and 'secondary prevention or prevention'. The explode function was used for applicable Medical Subject Headings, and truncation expanded the search for terms with unique endings. The search terms were refined for different databases. No limits were placed on years of publication to prevent restricting searches.

The combined searches resulted in the retrieval of a total of 1465 citations; Figure 1 presents the combined totals for the two searches. In keeping with the iterative nature of Arksey \& O'Malley's (2005) framework, becoming familiar with retrieved literature enabled a determination of study selection criteria. To determine study selection criteria, retrieved studies were reviewed for any discrepancies related to terminology used to define the population or intervention. A method to eliminate studies included developing inclusion and exclusion criteria based on the research question. In a scoping study, 


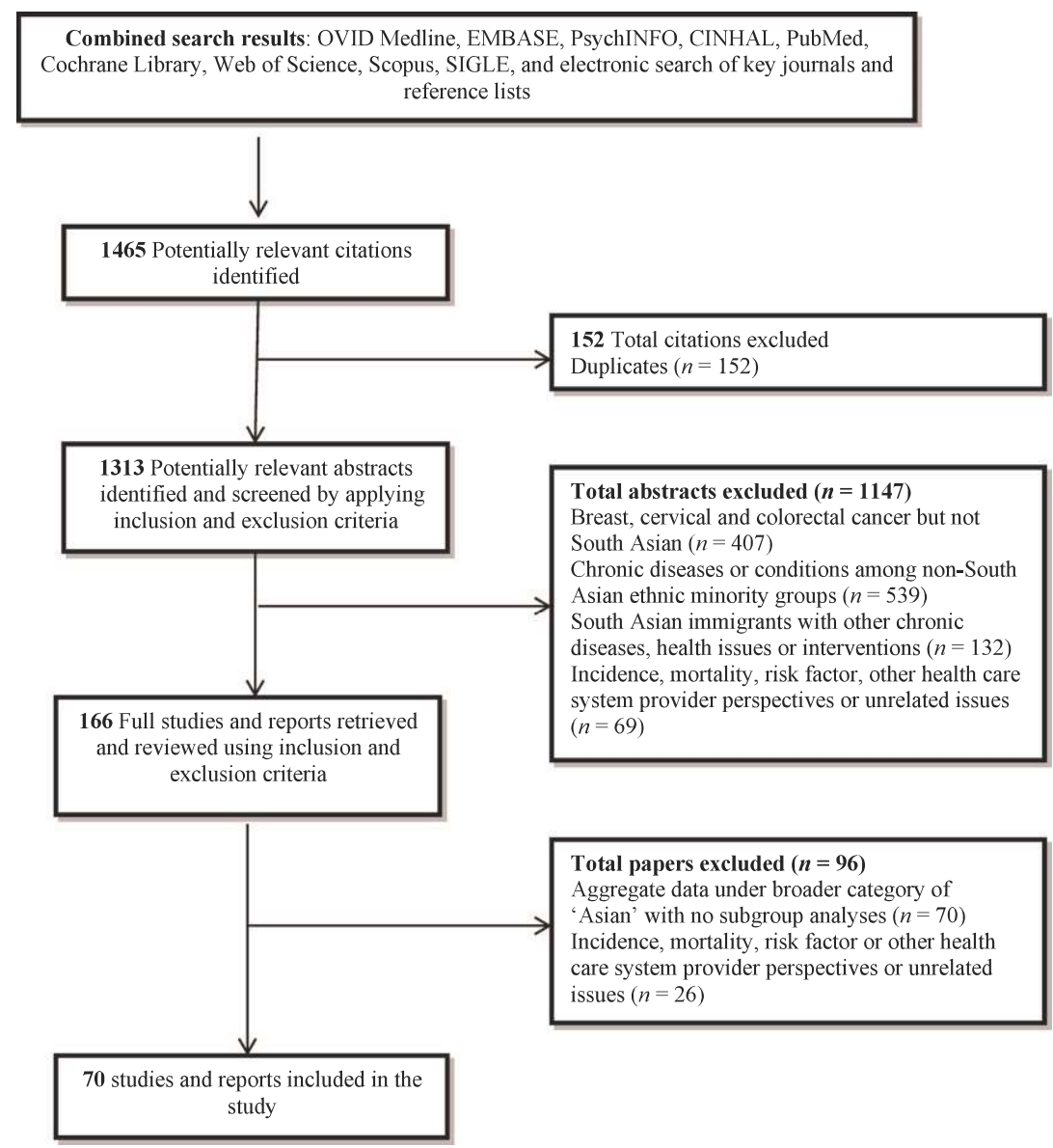

Figure 1 Scoping study flow diagram.

this is done posthoc to become familiar with the available literature. The aim of applying relevancy criteria to studies was to ensure that selected studies for review focused on answering the research question (Arksey \& O'Malley 2005).

Inclusion and exclusion criteria incorporated type of study, target population and type of intervention. Studies and reports were included if they: (i) employed quantitative and qualitative methods, were published in English between 1994 and 2014 and were accessible; (ii) included samples of SA immigrant men and women who resided in the UK, US and Canada; and (iii) investigated factors related to breast, cervical and colorectal cancer screening services. Studies were excluded if they reported on breast, cervical and colorectal cancers, but did not include SA immigrants; discussed chronic diseases or health issues among non-SA ethnic minorities; reported on incidence, mortality, risk factors of all three cancers; centred on healthcare system providers' perspectives or other unrelated issues; or aggregated data under a broader category of 'Asian' with no clear distinction of SA immigrants or subgroups. RefWorks (2.0) was used to organise and manage literature searches and retrieved citations.

An additional step was to contact practitioners and preventive healthcare providers to identify additional references, unpublished reports or to gain insight into the topic area (Arksey \& O'Malley 2005). Informal contact was made with public health practitioners working in the field and key organisations such as Cancer Care Ontario for any additional unpublished reports.

The primary reviewer independently applied inclusion/exclusion criteria to all abstract citations during abstract review. If relevancy was difficult to ascertain from an abstract, the full text article was retrieved. The primary reviewer read all potential full-text papers. In the case of ambiguity of a particular study or report, team members consulted and discussed whether a paper met criteria for inclusion. A total of 70 studies and reports met inclusion criteria (Figure 1). 
Selected studies were reviewed, data were extracted and organised, and relevant information was charted under the following headings: (i) author, year of publication, study location and topic area; (ii) study design and purpose; (iii) study population and setting; (iv) methods; and (v) findings. A narrative approach was used to capture dominant and important themes that emerged. Thematic analysis was used to focus attention on context and commonalities across included studies and reports, which was guided by the original research question (Mays et al. 2005).

\section{Findings}

The 70 included articles covered 20 years from 1994 to 2014. Studies and reports were primarily descriptive or exploratory, and focused mainly on breast and cervical cancer screening among SA immigrant women (Table 1). A numerical summary was created to provide an overview of the distribution of studies by geographical location, type of cancer screening, research methods and main topic areas (Table 2). In the following paragraphs, the descriptive findings will be presented and include study design, samples, the type of screening and country of origin. Thereafter, the thematic analysis of findings will be discussed, including the four main themes emerging across all included studies.

The majority of studies and reports $(66 \%, n=46)$ were cross-sectional, used surveys, examined selfreport screening rates, barriers and factors associated with cancer screening participation. Cross-sectional studies that included different ethno-cultural groups often had small samples of SA immigrants or SA immigrant subgroups. Almost one-third (29\%, $n=20)$ of studies used qualitative designs with data collection methods of focus groups, interviews and concept mapping. The remaining four studies (5\%) used mixed-methods or pre/post intervention design. The majority of qualitative studies $(60 \%, n=12 / 20)$ examined female cancer screening or beliefs and barriers to healthcare services including cancer screening. Of the remaining studies, one interviewed breast cancer patients and their spouses (Karbani et al. 2011), one did not clarify male and female participant numbers (Randhawa, \& Owens, 2004), one had almost equivalent male and female samples (Lee et al. 2010b) and five had greater female than male participants (Thomas et al. 2005, Asanin \& Wilson 2007, Austin et al. 2009, Lobb et al. 2013, Gesink et al. 2014). Two mixedmethods studies also used qualitative design with one including female samples only (Meana et al. 2001b) and the other conducted with both female and male samples (Szczepura et al. 2003). Fifteen (21\%) studies included colorectal cancer screening and were undertaken in the UK, US and Canada; of these, 14 included males and females. In colorectal cancer screening studies, findings varied by test procedures investigated. Four studies from the UK and Canada qualitatively examined SA immigrants' perspectives on beliefs, barriers and reasons for colorectal cancer screening (Szczepura et al. 2003, Austin et al. 2009, Lobb et al. 2013, Gesink et al. 2014).

\section{Thematic analysis}

Through charting and thematic analysis, four dominant recurring and relevant themes were identified: (i) beliefs and attitudes towards cancer and screening including sub-themes of family as central, holistic healthcare, fatalism, screening not necessary and emotion-laden perceptions; (ii) lack of knowledge of cancer and screening related to not having heard about cancer and its causes, or lack of awareness of screening, its rationale and how to access services; (iii) barriers to access centred on individual and structural barriers to cancer prevention or screening services; and (iv) gender differences in screening comprised of rates and factors associated with screening uptake. See Table 3 for themes and subthemes, and information on gender distribution of studies.

\section{Theme 1: beliefs and attitudes}

The first two sub-themes emerged as important contributors to SA immigrants' beliefs of cancer and cancer screening uptake providing insights into the sociocultural context and use of health services including screening, whereas the subsequent three sub-themes revealed the reasons for which SA immigrants did not participate in cancer screening.

Family as central. Common beliefs included a strong sense of family cohesiveness demonstrating honour, respect and dependence (Bottorff et al. 1998, 2001b, Choudhry 1998, Oelke \& Vollman 2007). Respecting and honouring family were maintained by not discussing sensitive female health-related issues such as cervical or breast cancers within the family (Bottorff et al. 2001b, Banning \& Hafeez 2010) or with others in the community (Bottorff et al. 1998, 2001b, Choudhry 1998, Meana et al. 2001a, Oelke \& Vollman 2007). The inability to discuss relevant health issues with others (Bottorff et al. 1998, Meana et al. 2001a) limited conversations that may have served to increase awareness of recommended preventive 


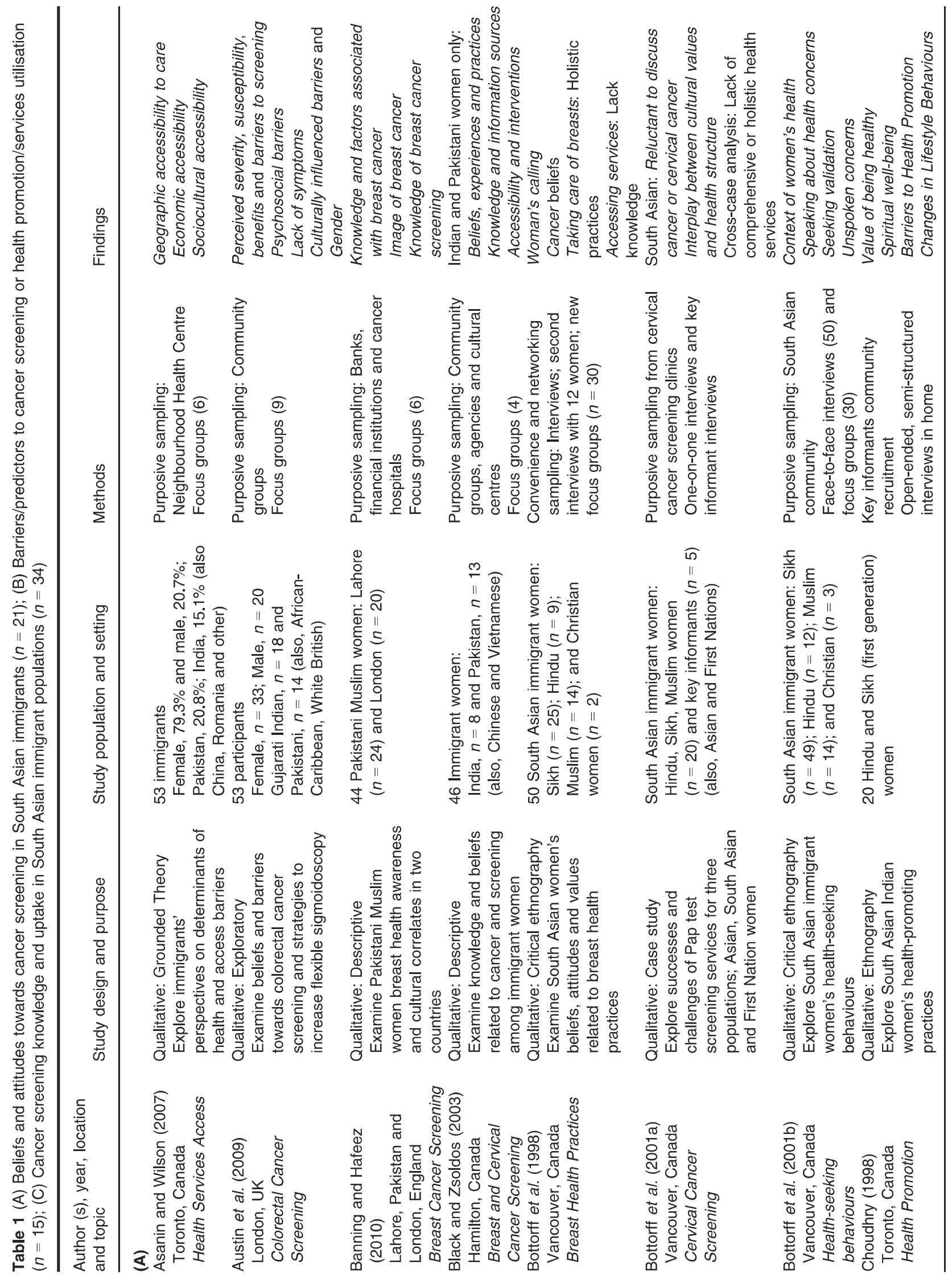




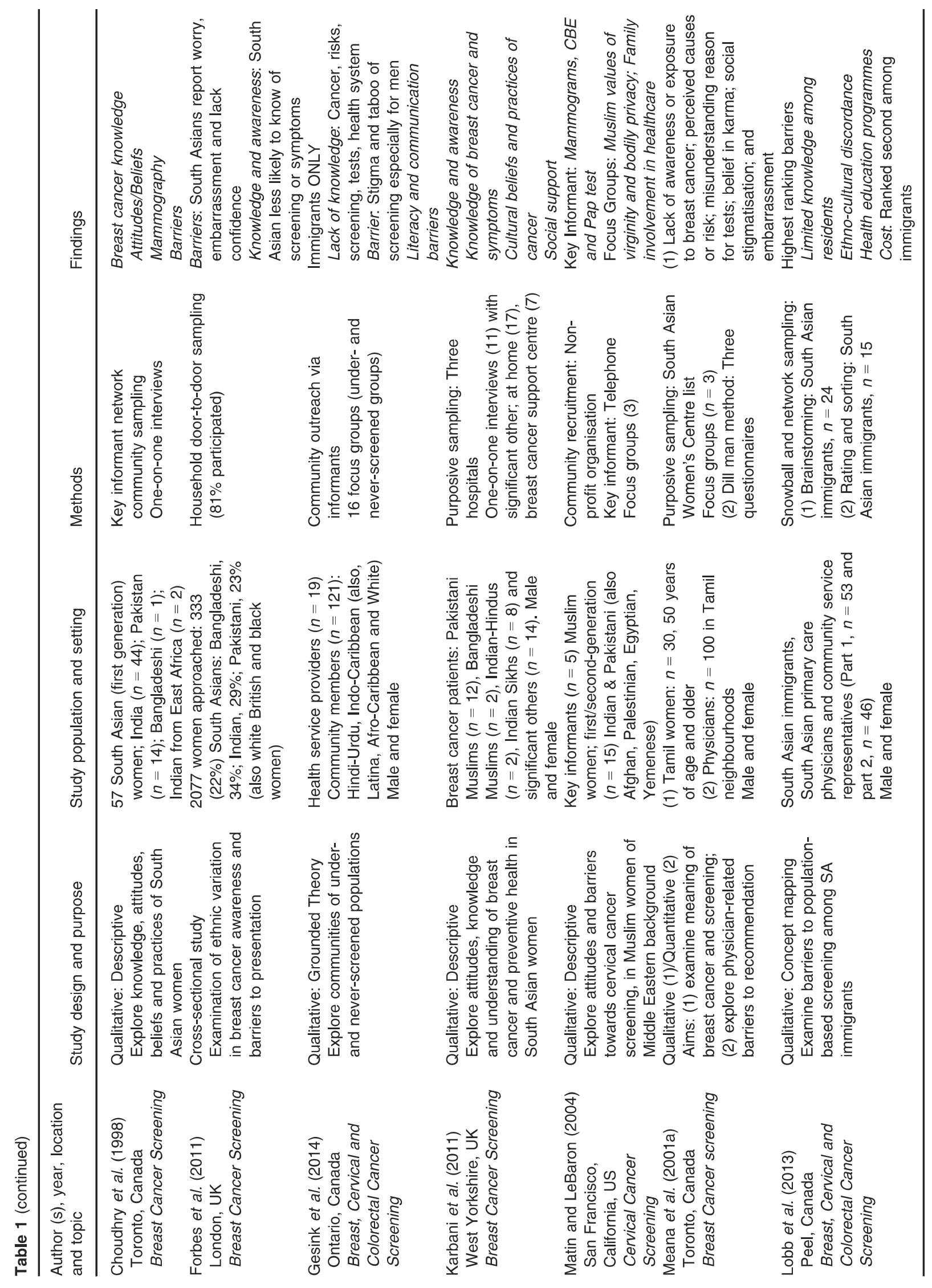




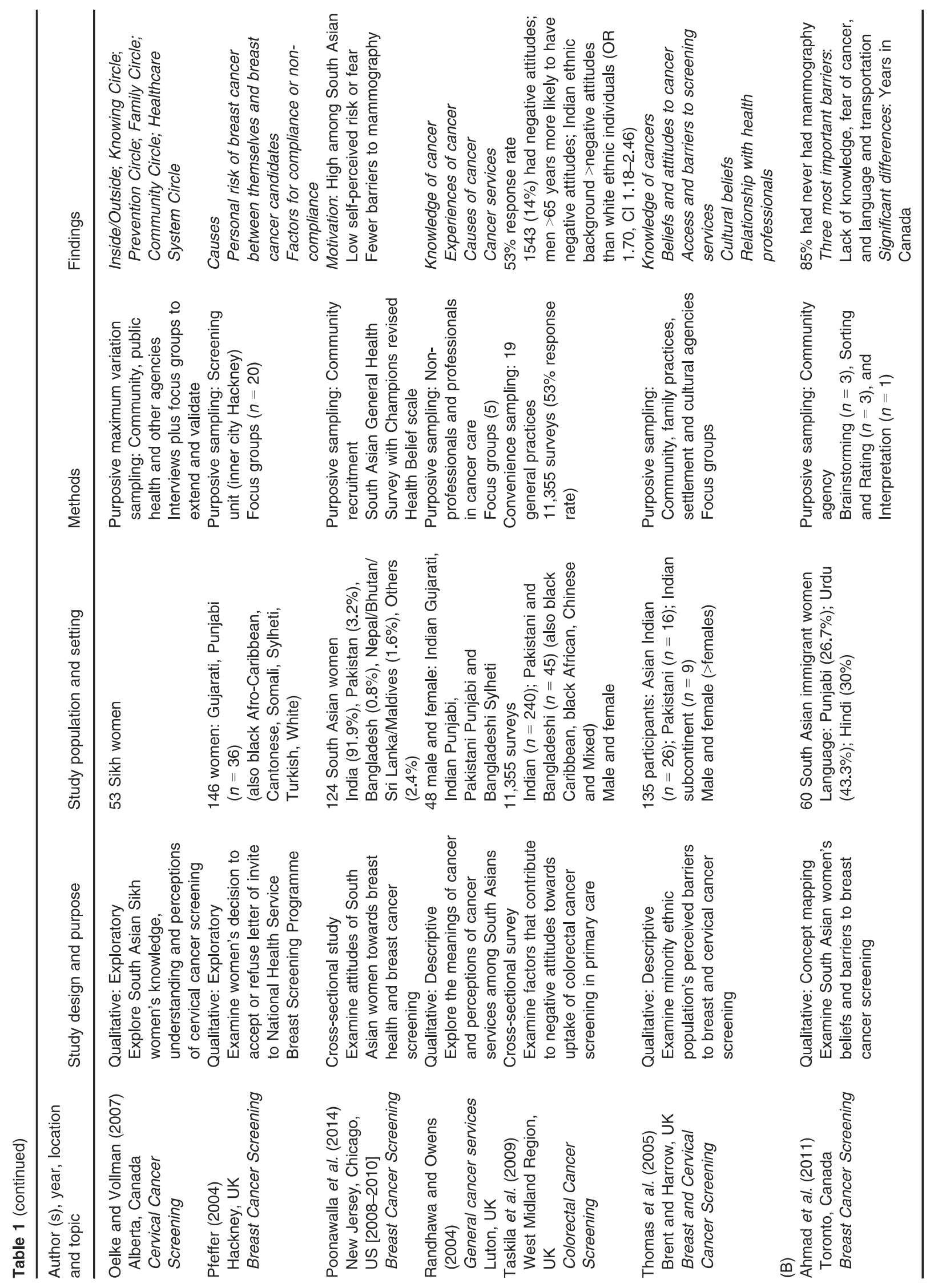




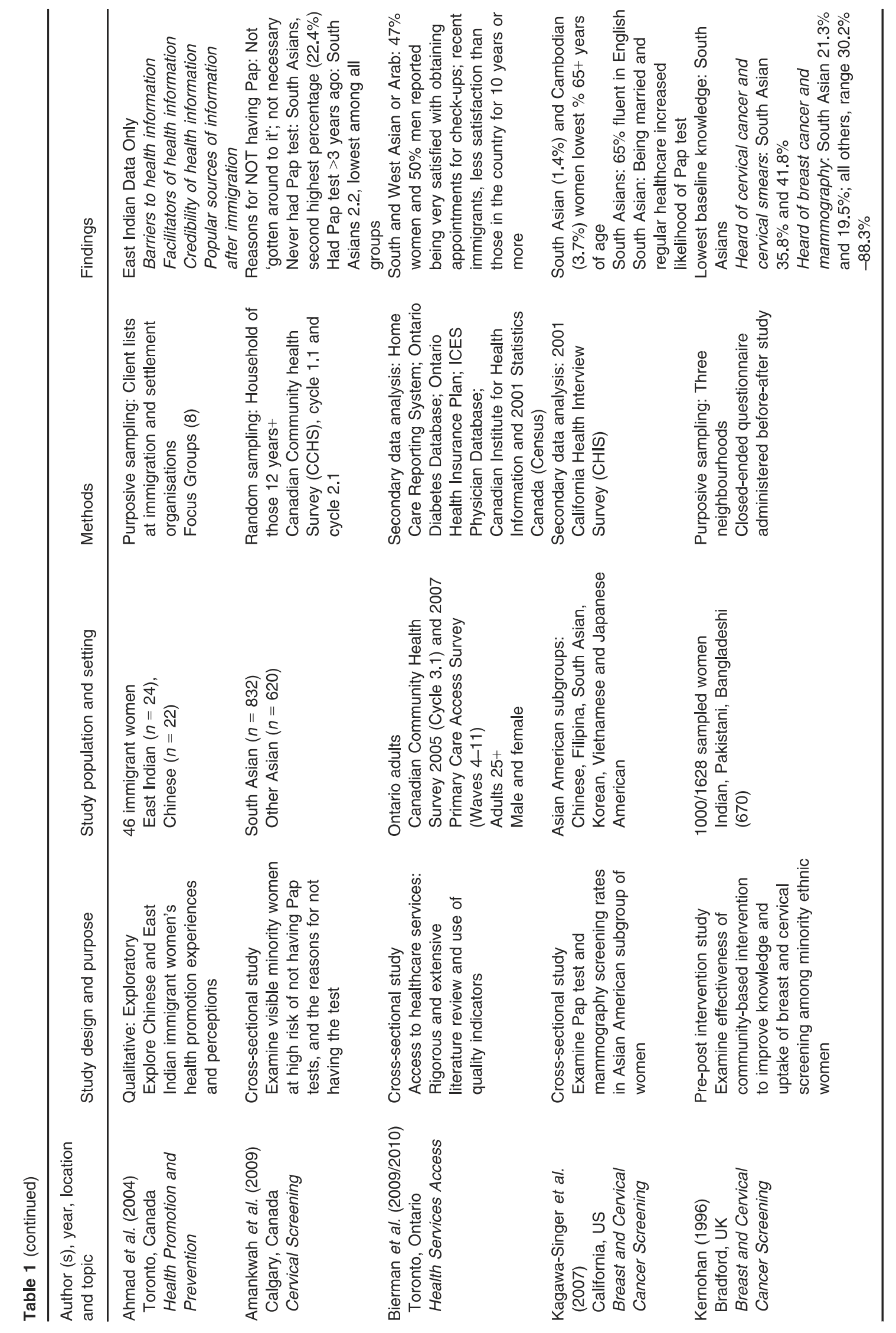




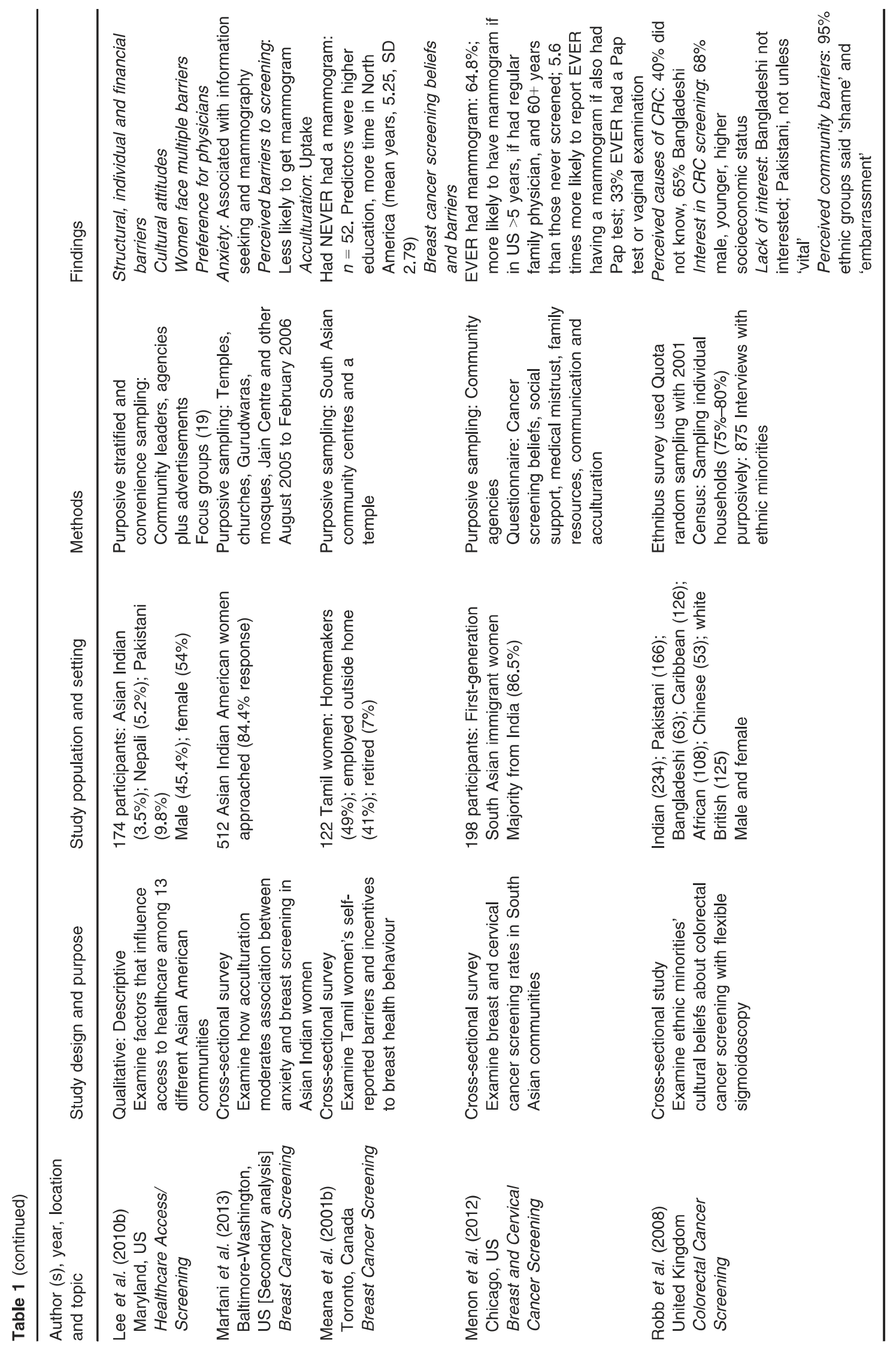




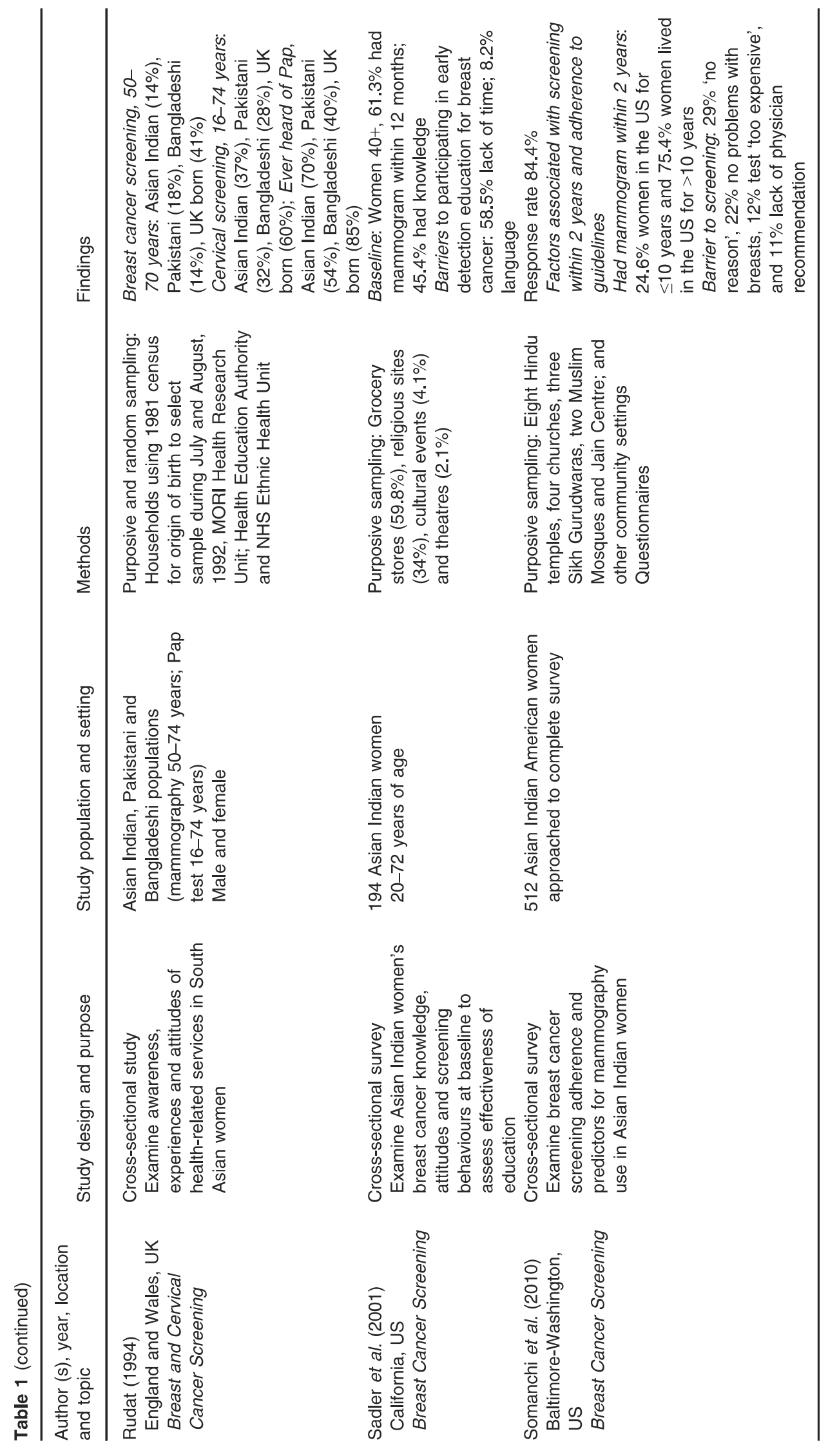




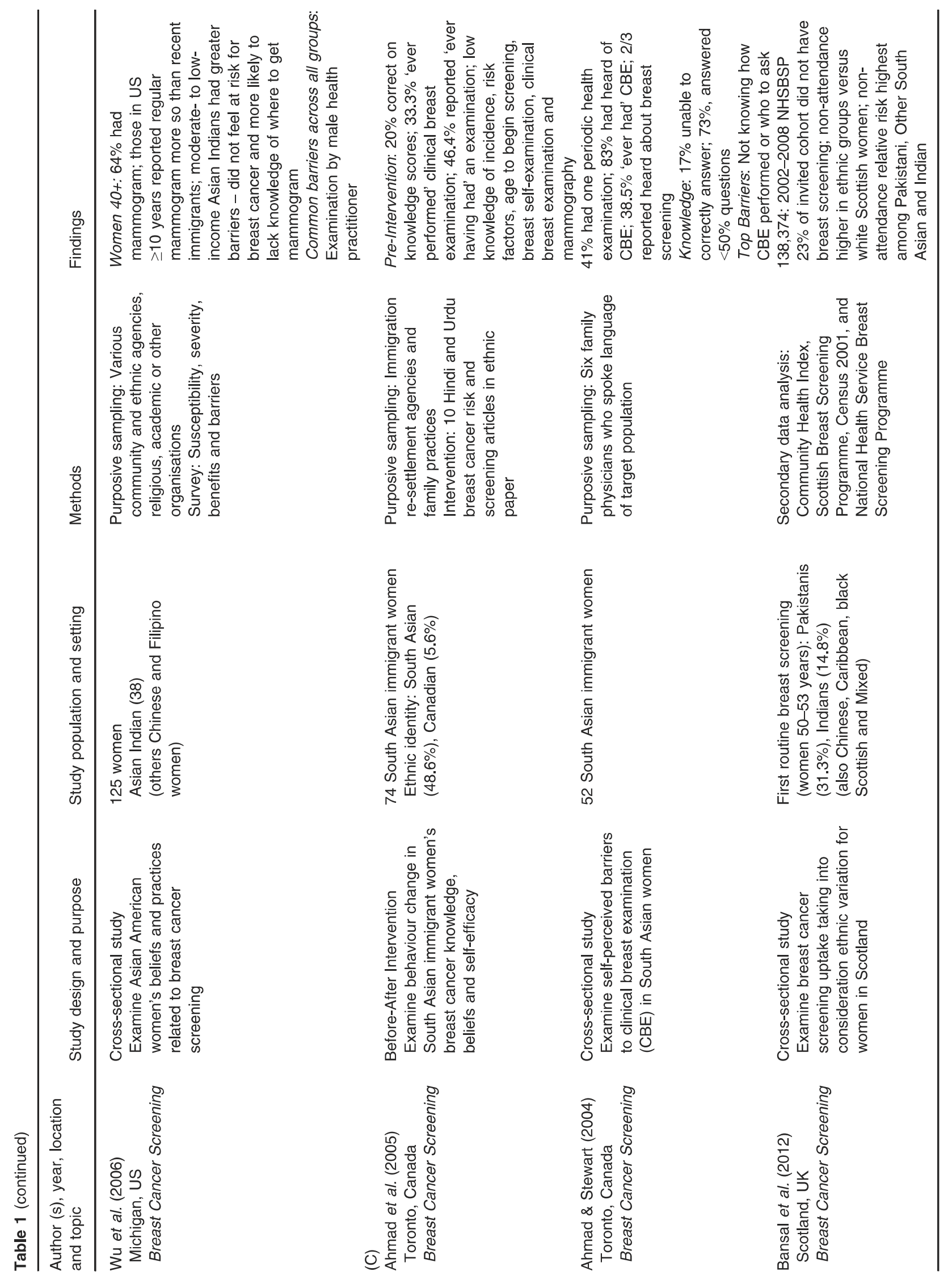




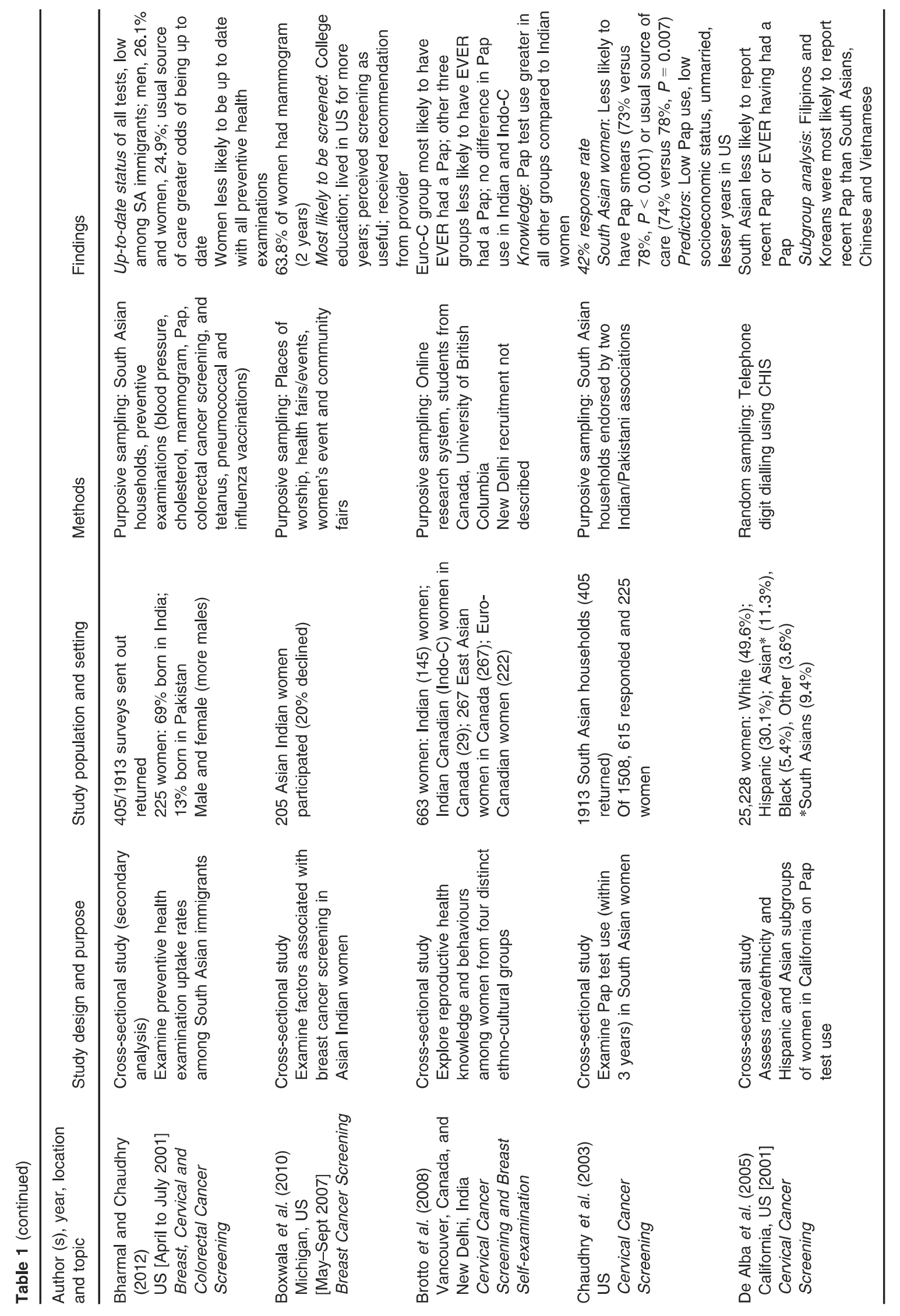




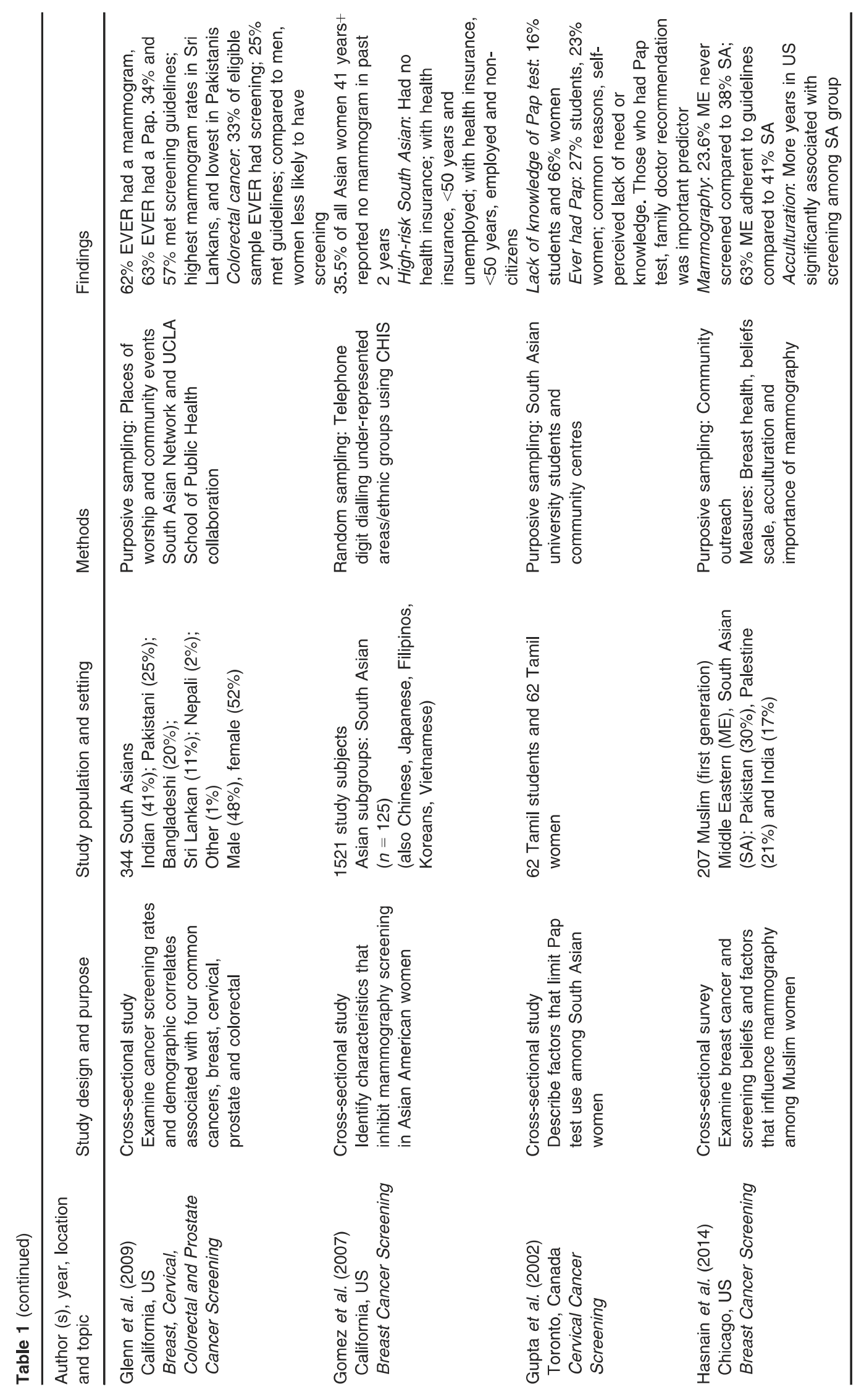




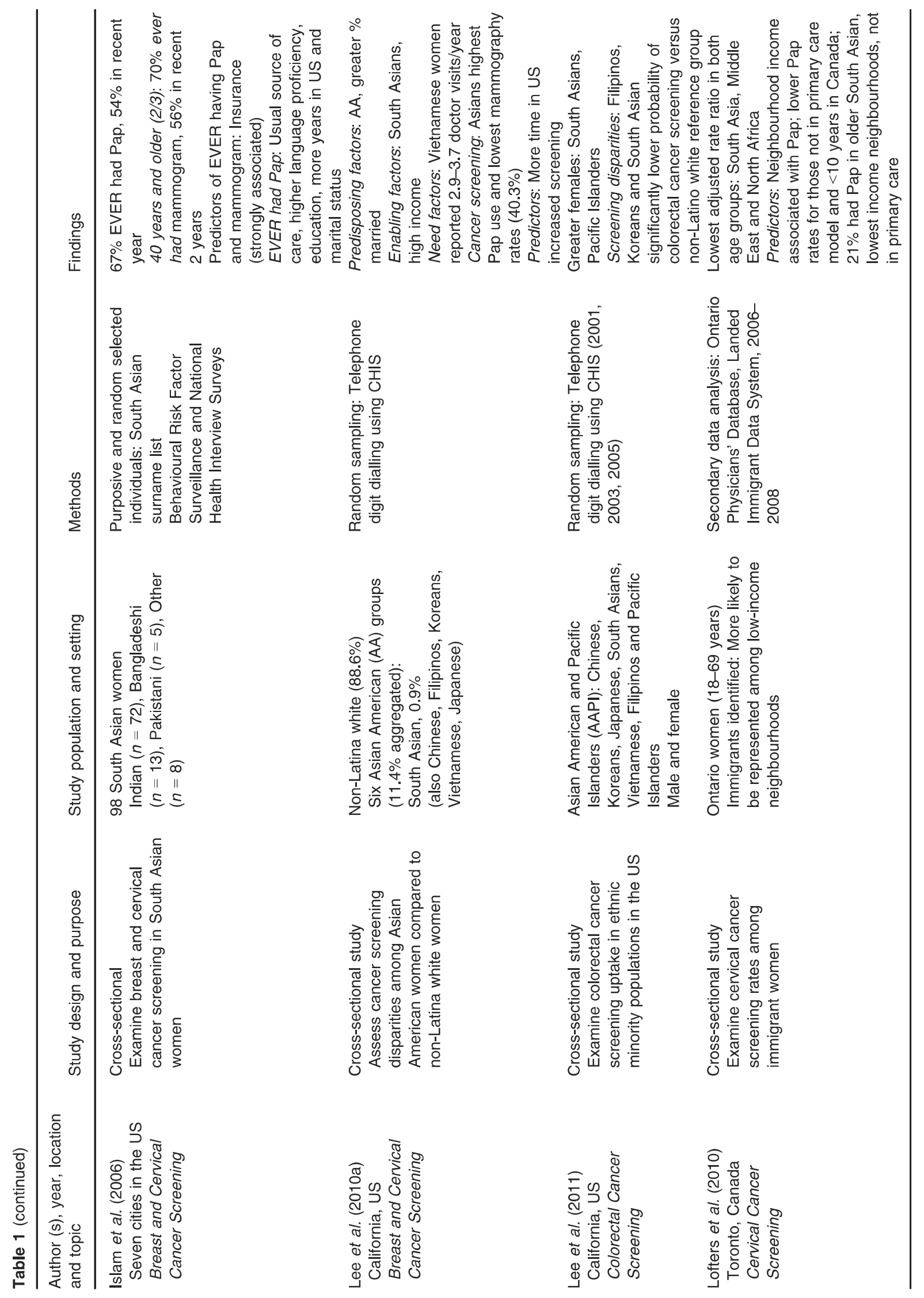




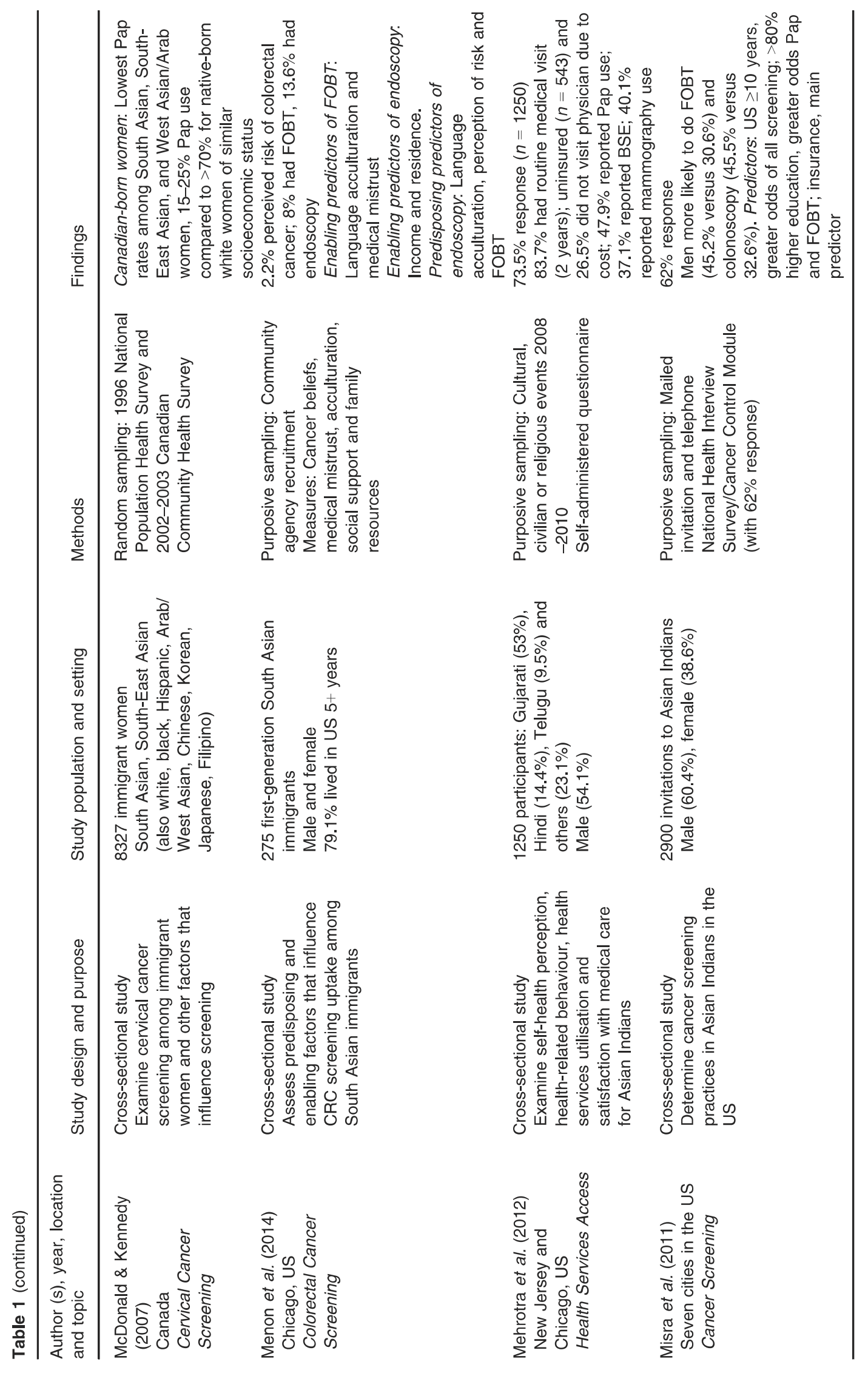




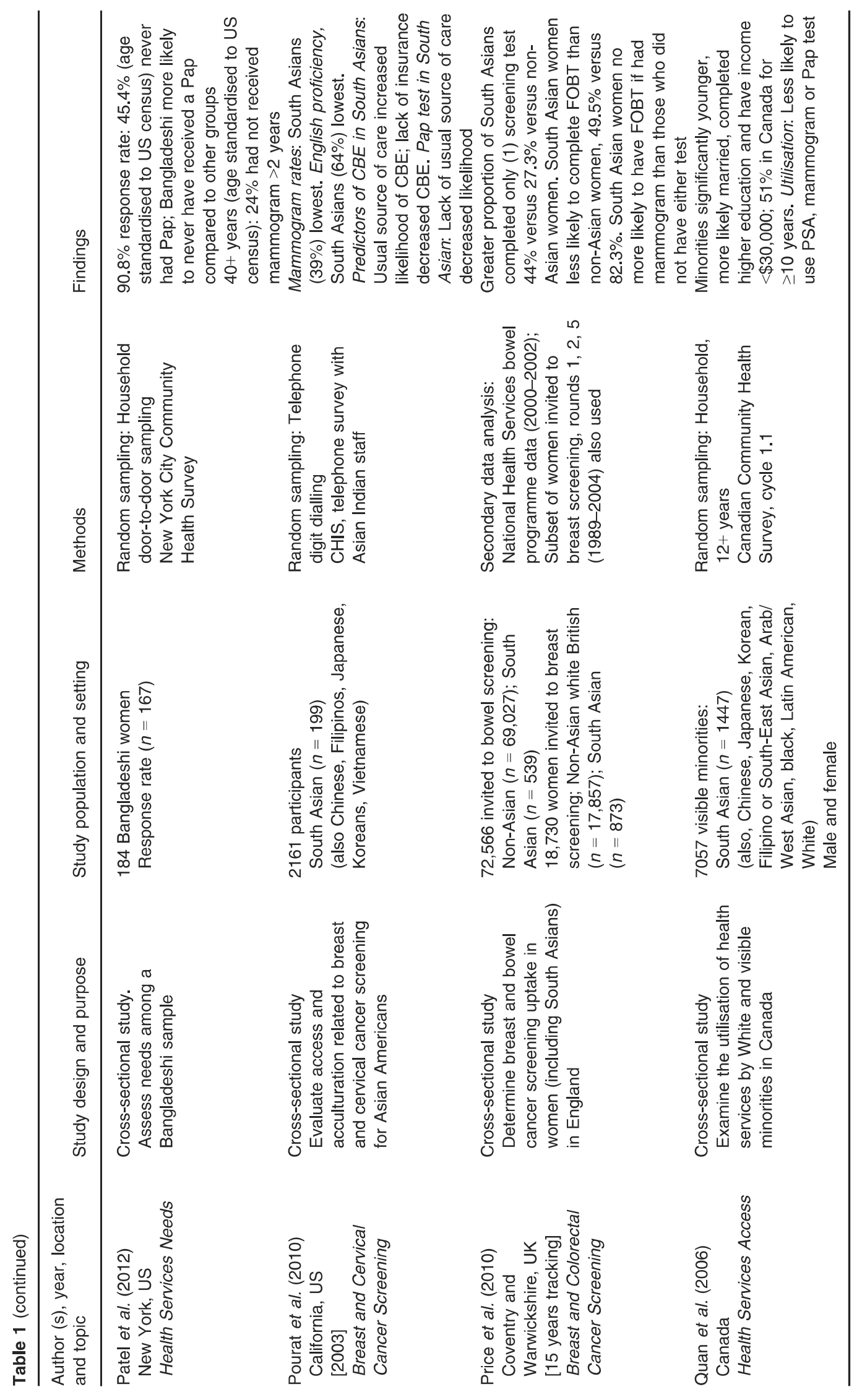




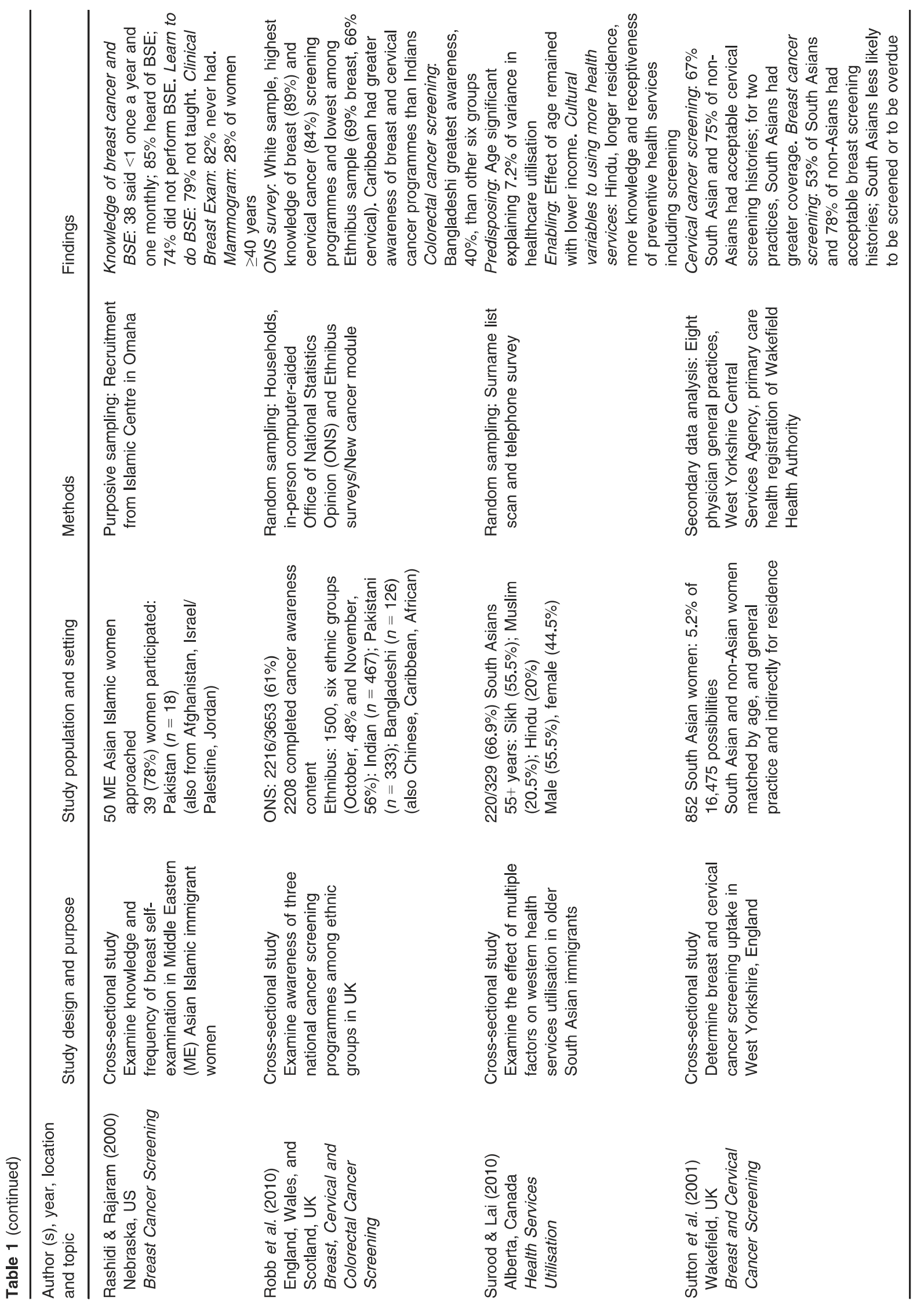




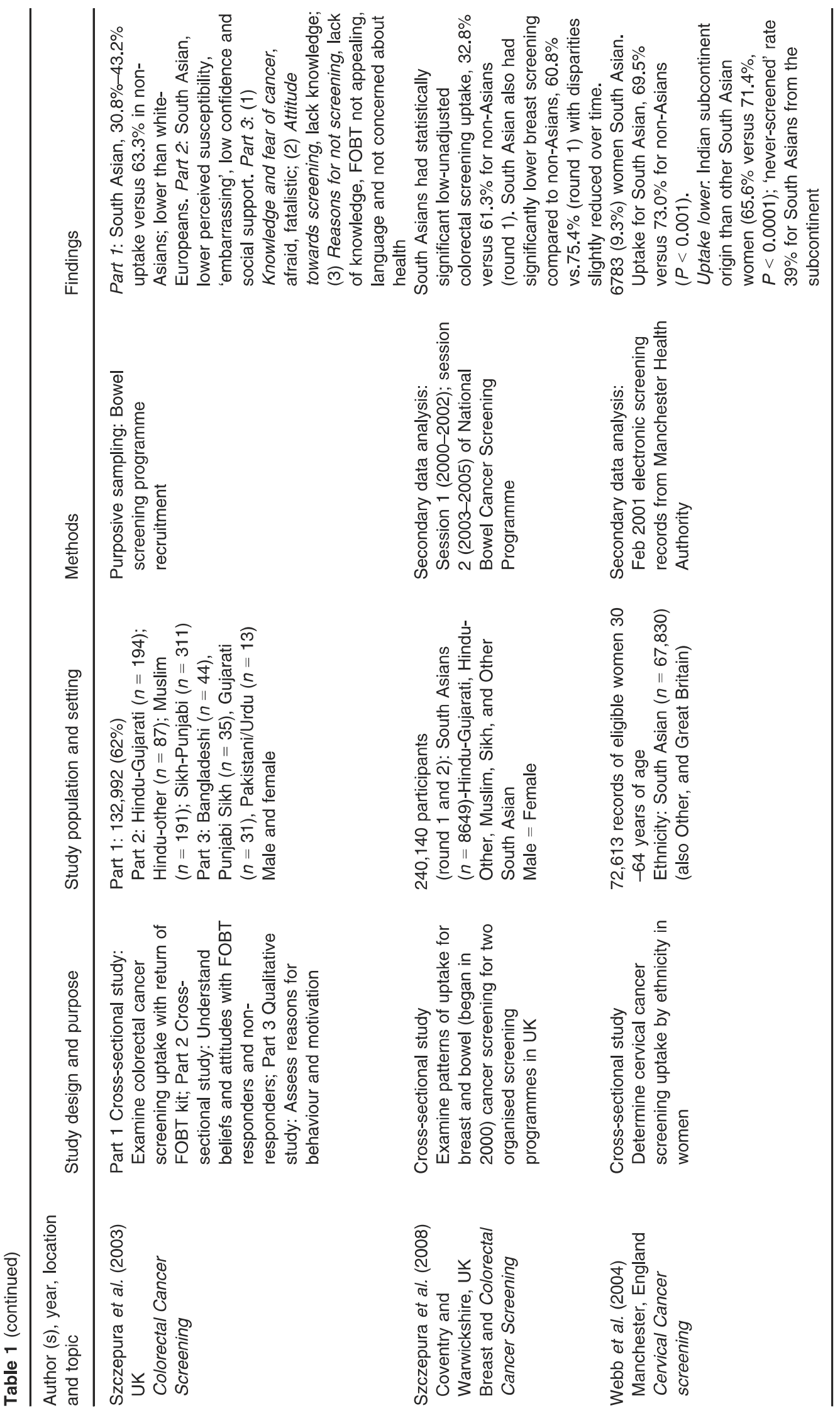




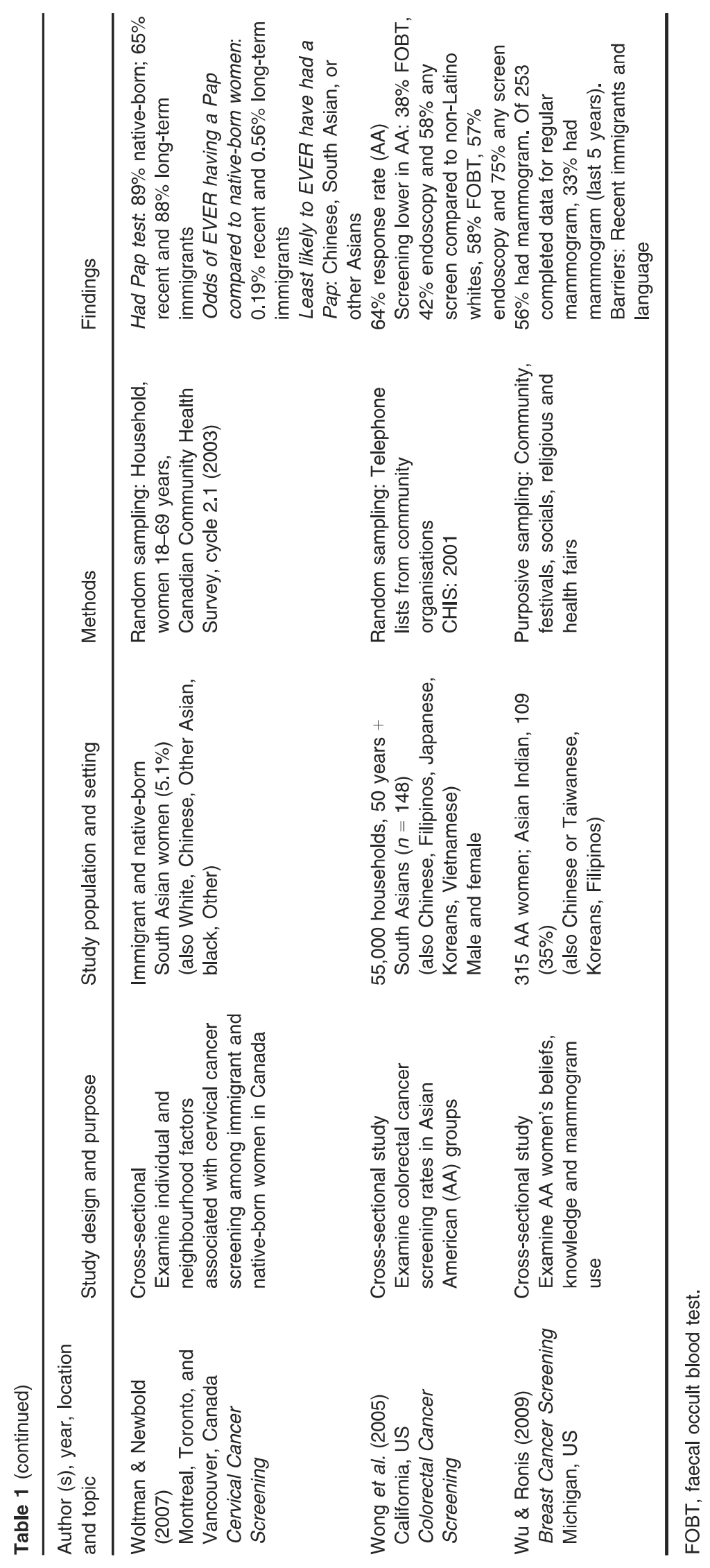


Table 2 Scoping study: numerical summary of research studies

\begin{tabular}{|c|c|c|c|}
\hline Geography & UK & US & Canada \\
\hline \multicolumn{4}{|l|}{ Screening or services } \\
\hline Colorectal screening & 4 & 3 & 0 \\
\hline Breast screening & 5 & 10 & 7 \\
\hline Cervical screening & 1 & 3 & 7 \\
\hline Breast and cervical screening & 4 & 5 & 2 \\
\hline Breast, cervical and colorectal screening & 1 & 3 & 2 \\
\hline Breast and colorectal screening & 2 & 0 & 0 \\
\hline Health promotion/services provision & 1 & 3 & 7 \\
\hline \multicolumn{4}{|l|}{ Research methods } \\
\hline Quantitative: cross-sectional surveys & 10 & 25 & 11 \\
\hline $\begin{array}{l}\text { Qualitative: focus group/one-on-one } \\
\text { interviews }\end{array}$ & 6 & 2 & 12 \\
\hline $\begin{array}{l}\text { Other: mixed methods/review/intervention } \\
\text { study }\end{array}$ & 2 & 0 & 2 \\
\hline \multicolumn{4}{|l|}{ General topic area } \\
\hline $\begin{array}{l}\text { Beliefs and attitudes (Table } 1 \mathrm{~A}) \\
\text { barriers/predictors to utilisation } \\
\text { (Table 1B) }\end{array}$ & 11 & 9 & 16 \\
\hline Knowledge and uptake (Table 1C) & 7 & 18 & 9 \\
\hline Total studies & 18 & 27 & 25 \\
\hline
\end{tabular}

health practices (Bottorff et al. 2001b, Oelke \& Vollman 2007). Maintaining privacy was another important concern, especially when accessing health services in smaller communities (Oelke \& Vollman 2007).

In contrast, families could play an important role in validating concerns, and providing advice and recommendations (Oelke \& Vollman 2007). The head of the household or a close friend sometimes provided advice on health issues or consulted on whether it was necessary to seek out physician advice (Bottorff et al. 1998, 2001b, Ahmad et al. 2004, Oelke \& Vollman 2007, Banning \& Hafeez 2010). Family support to access healthcare was especially important when women did not speak English (Choudhry 1998, Ahmad et al. 2011). A lack of informal support networks or extended family increased a SA immigrant woman's dependence on family members when accessing health services (Choudhry 1998, Thomas et al. 2005). For some SA Muslim women, family played a role in assuring cultural values were maintained with respect to western healthcare practices, for example, the recommendation of a Pap test for an unmarried woman went against Muslim beliefs and values (Matin \& LeBaron 2004). Alternatively, some SA immigrants' immediate family and relatives encouraged them to have screening (Oelke \& Vollman 2007, Karbani et al. 2011).

Some SA immigrant women lived in patriarchal families, where their primary role was to meet family obligations including care-giving, homemaking and/ or contributing by working outside the home (Bot- torff et al. 1998, 2001b, Oelke \& Vollman 2007). Family needs could supersede personal needs (Oelke \& Vollman 2007). In other situations, SA immigrant women believed that they were not to burden their family; and so, good health maintenance was required to fulfil their obligations (Choudhry 1998, Bottorff et al. 2001b).

Holistic healthcare. A holistic approach to health was believed to be conducive to maintaining health among both SA immigrant men and women (Bottorff et al. 1998, Choudhry 1998, Asanin \& Wilson 2007). Some SA immigrant women reported the belief that health maintenance involved a balance between body, mind and spirit (Choudhry 1998, Bottorff et al. 2001b, Black \& Zsoldos 2003). Importance was placed on diet, physical activity, reduced levels of stress and relaxation (Bottorff et al. 1998, Choudhry 1998, Black \& Zsoldos 2003). Among SA immigrant women of higher socioeconomic status, greater motivation to take care of one's health and confidence with performing breast self-examination were associated with greater perceived benefits of mammography (Poonawalla et al. 2014).

In other situations, SA immigrants' healthcare encounters with physicians were not perceived to be holistic; rather, they were perceived to be rushed, impersonal and reserved, creating challenges and conflicts due to differing views of health (Asanin \& Wilson 2007, Lobb et al. 2013). In addition, SA immigrants believed that the health system was not respectful nor did it provide accommodation for the SA culture and traditional views of health, which created 'ethno-cultural discordance' (Lobb et al. 2013). A physician's lack of respect and sensitivity towards Muslim women's values of modesty and virginity were issues that did not align with cultural views among some SA immigrant women (Matin \& LeBaron 2004). SA immigrants also reported lower satisfaction with the routine health examination (Asanin \& Wilson 2007, Bierman et al. 2009/2010). The physician's role in promoting cancer screening was believed to be important for access to cancer screening tests (Lobb et al. 2013). As well, level of trust with doctors or other healthcare workers was an enabling predictor for faecal occult blood test (FOBT) uptake among SA immigrants (Menon et al. 2014). The discordance between what SA immigrants believed and what occurred in western health systems poses challenges in promoting health and cancer screening uptake.

Fatalism. Karma and destiny were directly linked to fatalistic beliefs, whereby cancer was one's destiny 
Table 3 Thematic analysis arising from studies and reports regarding cancer screening among South Asian (SA) immigrants

\begin{tabular}{|c|c|}
\hline Theme & Sub-theme \\
\hline $\begin{array}{l}\text { 1. Beliefs and } \\
\text { attitudes } \\
\text { Beliefs and } \\
\text { attitudes } \\
\text { towards } \\
\text { cancer } \\
\text { and screening }\end{array}$ & $\begin{array}{l}\text { Family as central } \\
\text { The cultural beliefs and values } \\
\text { associated with family such as } \\
\text { cohesiveness, respect and } \\
\text { honour were important findings } \\
\text { related to sociocultural context } \\
\text { of SA immigrants } \\
\text { Holistic healthcare } \\
\text { The perception that maintaining } \\
\text { health also occurs informally, } \\
\text { and involves lifestyle balance } \\
\text { (diet, physical activity, rest, } \\
\text { reduced stress) } \\
\text { Fatalism } \\
\text { The views associated with } \\
\text { cancer emerged as a strong } \\
\text { belief that it was out of } \\
\text { individual control and led to } \\
\text { death } \\
\text { Screening not necessary } \\
\text { The low self-perceived risk that } \\
\text { screening was only indicated for } \\
\text { those at risk, or those who had } \\
\text { symptoms }\end{array}$ \\
\hline & $\begin{array}{l}\text { Emotion-laden perceptions } \\
\text { Negative emotional states were } \\
\text { reasons for not engaging in } \\
\text { cancer screening }\end{array}$ \\
\hline
\end{tabular}

\section{Lack of knowledge}

Reasons for not engaging in cancer screening included limited knowledge of cancer type, the causes of cancer, awareness or types of screening tests, and access points to obtain screening

3. Barriers to
access
Individualised or
systematic reasons
that impede the ability
to access cancer
screening

3. Barriers to Individualised or systematic reasons to access cancer

\author{
Individual barriers \\ The personal and individual \\ factors that inhibit individuals \\ from accessing cancer \\ screening, such as language, \\ social support, time, money and \\ transportation \\ Structural barriers \\ The systemic factors inherent in \\ the way health services are \\ organised that limit access to \\ cancer screening, such as \\ physician gender, culture or \\ recommendation
}

Study distribution and references

11 studies: one included samples of both genders

Bottorff et al. (1998), Choudhry (1998), Bottorff et al. (2001b), Meana et al. (2001a), Ahmad et al. (2004), Matin \& LeBaron (2004), Thomas et al. (2005), Oelke \& Vollman (2007), Banning \& Hafeez (2010), Ahmad et al. (2011), Karbani et al. (2011)

10 studies: four included samples of both genders

Bottorff et al. (1998), Choudhry (1998), Bottorff et al. (2001b), Black \& Zsoldos (2003), Matin \& LeBaron (2004), Asanin \& Wilson (2007), Bierman et al. (2009/2010), Lobb et al. (2013), Menon et al. (2014), Poonawalla et al. (2014)

10 studies: six included samples of both genders

Bottorff et al. (1998), Choudhry (1998), Meana et al. (2001a), Black \& Zsoldos (2003), Szczepura et al. (2003), Pfeffer (2004), Randhawa \& Owens (2004), Thomas et al. (2005), Karbani et al. (2011), Gesink et al. (2014)

14 studies: six included samples of both genders

Rudat (1994), Bottorff et al. (1998), Sadler et al. (2001), Szczepura et al. (2003), Pfeffer (2004), Thomas et al. (2005), Wu et al. (2006), Oelke \& Vollman (2007), Robb et al. (2008), Amankwah et al. (2009), Austin et al. (2009), Lobb et al. (2013), Menon et al. (2014), Poonawalla et al. (2014)

21 studies: seven included samples of both genders

Rudat (1994), Bottorff et al. (1998), Choudhry et al. (1998), Bottorff et al. (2001a), Meana et al. (2001a), Meana et al. (2001b), Sadler et al. (2001), Black \& Zsoldos (2003), Szczepura et al. (2003), Ahmad \& Stewart (2004), Pfeffer (2004), Thomas et al. (2005), Oelke \& Vollman (2007), Robb et al. (2008), Austin et al. (2009), Taskila et al. (2009), Banning \& Hafeez (2010), Ahmad et al. (2011), Forbes et al. (2011), Lobb et al. (2013), Poonawalla et al. (2014)

23 studies: 7 included samples of both genders

Rudat (1994), Bottorff et al. (1998), Choudhry et al. (1998), Rashidi \& Rajaram (2000), Bottorff et al. (2001a), Meana et al. (2001b), Gupta et al. (2002), Szczepura et al. (2003), Ahmad et al. (2004), Ahmad \& Stewart (2004), Ahmad et al. (2005), Wu et al. (2006), Oelke \& Vollman (2007), Brotto et al. (2008), Robb et al. (2008), Austin et al. (2009), Wu \& Ronis (2009), Banning \& Hafeez (2010), Robb et al. (2010), Forbes et al. (2011), Karbani et al. (2011), Lobb et al. (2013), Gesink et al. (2014)

18 studies: nine included samples of both genders

Kernohan (1996), Bottorff et al. (1998), Meana et al. (2001b), Sadler et al. (2001), Szczepura et al. (2003), Ahmad et al. (2004), Matin \& LeBaron (2004), Thomas et al. (2005), Asanin \& Wilson (2007), Oelke \& Vollman (2007), Austin et al. (2009), Szczepura et al. (2008), Wu \& Ronis (2009), Lee et al. (2010b), Ahmad et al. (2011), Karbani et al. (2011), Lobb et al. (2013), Gesink et al. (2014) 23 studies: 10 included samples of both genders

Rudat (1994), Bottorff et al. (1998), Bottorff et al. (2001a), Meana et al. (2001a), Gupta et al. (2002), Black \& Zsoldos (2003), Pfeffer (2004), De Alba et al. (2005), Thomas et al. (2005), Wong et al. (2005), Islam et al. (2006), Asanin \& Wilson (2007), Gomez et al. (2007), Oelke and Vollman (2007), Glenn et al. (2009), Boxwala et al. (2010), Lee et al. (2010b), Pourat et al. (2010), Somanchi et al. (2010), Karbani et al. (2011), Misra et al. (2011), Mehrotra et al. (2012), Lobb et al. (2013) 
Table 3 (continued)

\begin{tabular}{|c|c|}
\hline Sub-theme & Study distribution and references \\
\hline 4. Gender differences & 39 studies: 10 included samples of both genders \\
\hline $\begin{array}{l}\text { The distinct factors that affect uptake of } \\
\text { cancer screening in SA men and SA women }\end{array}$ & $\begin{array}{l}\text { Rudat (1994), Kernohan (1996), Choudhry et al. (1998), Rashidi \& } \\
\text { Rajaram (2000), Meana et al. (2001a,b), Sutton et al. (2001), Gupta } \\
\text { et al. (2002), Chaudhry et al. (2003), Ahmad \& Stewart (2004), Webb } \\
\text { et al. (2004), De Alba et al. (2005), Wong et al. (2005), Islam et al. } \\
\text { (2006), Quan et al. (2006), Gomez et al. (2007), McDonald \& Kennedy } \\
\text { (2007), Kagawa-Singer et al. (2007), Woltman \& Newbold (2007), Brotto } \\
\text { et al. (2008), Szczepura et al. (2008), Glenn et al. (2009), Wu \& Ronis } \\
\text { (2009), Amankwah et al. (2009), Boxwala et al. (2010), Lee et al. } \\
\text { (2010a), Lofters et al. (2010), Pourat et al. (2010), Price et al. (2010), } \\
\text { Somanchi et al. (2010), Surood \& Lai (2010), Misra et al. (2011), Bansal } \\
\text { et al. (2012), Bharmal and Chaudhry (2012), Mehrotra et al. (2012), } \\
\text { Menon et al. (2012), Patel et al. (2012), Hasnain et al. (2014), Marfani } \\
\text { et al. (2013), Menon et al. (2014) }\end{array}$ \\
\hline
\end{tabular}

determined by God (Choudhry 1998, Meana et al., 2001a, Black \& Zsoldos 2003, Szczepura et al. 2003). Some SA immigrants believed that cancer was 'incurable' (Bottorff et al. 1998, Randhawa \& Owens 2004), and not a disease that could be prevented or controlled (Meana et al., 2001a, Black \& Zsoldos 2003). Beliefs associated with developing cancer included negative lifestyle behaviours such as promiscuity and physical inactivity; retribution for past sins; and a form of punishment (Meana et al. 2001a, Black \& Zsoldos 2003, Pfeffer 2004). Cancer was not to be discussed with family, relatives or the community (Bottorff et al. 1998, Meana et al. 2001a, Thomas et al. 2005, Karbani et al. 2011), and for some, avoiding talking about cancer was a way to prevent affliction with the disease (Meana et al. 2001a, Thomas et al. 2005). In addition, the stigma associated with a cancer diagnosis had the potential to damage a family's reputation (Karbani et al. 2011). In particular, some male SA immigrants avoided screening because of the stigma or taboo associated with seeing a doctor to discuss cancer screening (Gesink et al. 2014).

Screening not necessary. Some SA immigrants had low self-perceived risk of cancer. This was associated with SA immigrants' experiences in their country of origin and the belief that western-born populations were at higher risk for breast, cervical or colorectal cancers. For some SA immigrants, breast cancer was seen as a 'white woman's disease' (Bottorff et al. 1998) because they did not breast feed their children (Pfeffer 2004); screening was for younger women (Thomas et al. 2005); or cancer was not a risk for women of their culture (Rudat 1994, Wu et al. 2006; Poonawalla et al. 2014). Others believed that colorectal cancer was a predominantly male disease (Austin et al. 2009) or not a risk for them (Menon et al. 2014). A lack of symptoms was another reason for believing that screening was not required (Bottorff et al. 1998, Szczepura et al. 2003, Oelke \& Vollman 2007, Robb et al. 2008, Austin et al. 2009), as was the perception that screening was not important or a priority (Rudat 1994, Sadler et al. 2001, Amankwah et al. 2009, Lobb et al. 2013). For some, these beliefs may have stemmed from the lack of exposure to preventive healthcare in countries of origin (Oelke \& Vollman 2007).

Emotion-laden perceptions. A number of emotional states were cited as reasons for not engaging in cancer screening. Fear, pain and worry were common findings (Rudat, 1994, Bottorff et al. 1998, 2001a, Meana et al. 2001a,b, Black \& Zsoldos 2003, Szczepura et al. 2003, Ahmad \& Stewart 2004, Pfeffer 2004, Austin et al. 2009, Ahmad et al. 2011, Forbes et al. 2011, Lobb et al. 2013), as was shame and embarrassment (Rudat, 1994, Meana et al. 2001a,b, Sadler et al. 2001, Szczepura et al. 2003, Pfeffer 2004, Robb et al. 2008, Austin et al. 2009, Banning \& Hafeez 2010, Forbes et al. 2011, Lobb et al. 2013). Fear was associated with discussing cancer with the physician, finding cancer, going for screening alone and discomfort with test procedures (Meana et al. 2001b, Forbes et al. 2011, Lobb et al. 2013). A higher self-perceived susceptibility to cancer was also associated with enhanced fear (Poonawalla et al. 2014). Modesty, shyness, nervousness and physical discomfort all influenced decisionmaking about breast and cervical cancer screening (Bottorff et al. 1998, 2001a, Choudhry et al. 1998, Meana et al. 2001b, Black \& Zsoldos 2003, Pfeffer 2004, Oelke \& Vollman 2007, Banning \& Hafeez 
2010). Some SA immigrants lacked confidence in discussing symptoms with the physician (Forbes et al. 2011) or performing screening (Meana et al. 2001b, Thomas et al. 2005, Austin et al. 2009). SA immigrant men perceived colorectal cancer screening to be 'unpleasant', 'unhygienic' or not acceptable (Taskila et al. 2009), while other SA immigrants reported it was 'disgusting' (Szczepura et al. 2003). A reason for having colorectal cancer screening among both genders was 'peace of mind' or early detection before disease progression (Szczepura et al. 2003, Robb et al. 2008, Austin et al. 2009).

Theme 2: lack of knowledge

A lack of knowledge of cancer and screening was a common reason for low participation. Gaps in knowledge and awareness of the types of cancer, causes, individual risks and implications of the disease were frequently cited (Rashidi \& Rajaram 2000, Szczepura et al. 2003, Robb et al. 2008, Austin et al. 2009, Lobb et al. 2013). Likewise, there was a lack of knowledge of symptoms (Forbes et al. 2011), screening or the rationale (Rudat 1994, Choudhry et al. 1998, Rashidi \& Rajaram 2000, Meana et al. 2001b, Gupta et al. 2002, Szczepura et al. 2003, Ahmad et al. 2005, Oelke \& Vollman 2007, Brotto et al. 2008, Banning \& Hafeez 2010, Robb et al. 2010, Lobb et al. 2013) and benefits of screening (Bottorff et al. 2001a, Meana et al. 2001b). The screening procedures themselves posed challenges as some SA immigrants reported not knowing how to perform the tests, whom to ask about screening or lacked awareness of the screening programmes and locations in their community (Bottorff et al. 1998, Ahmad \& Stewart 2004, Wu et al. 2006, Wu \& Ronis 2009, Banning \& Hafeez 2010, Robb et al. 2010, Forbes et al. 2011, Karbani et al. 2011, Lobb et al. 2013). A lack of knowledge of health information, services and insurance processes (Ahmad et al. 2004, Gesink et al. 2014), and low priority placed on screening were also identified (Lobb et al. 2013).

Theme 3: barriers to access

Both individual and structural factors create barriers to accessing cancer screening.

Individual barriers. In addition to emotion-laden perceptions of screening presented earlier, language was a significant barrier to cancer screening access among non-English-speaking SA immigrants (Kernohan 1996, Bottorff et al. 1998, Sadler et al. 2001, Szczepura et al. 2003, Ahmad et al. 2004, Matin \& LeBaron 2004, Thomas et al. 2005, Asanin \& Wilson 2007, Oelke \& Vollman 2007, Austin et al. 2009, Wu
\& Ronis 2009, Lee et al. 2010b, Karbani et al. 2011). Low literacy levels were particularly an issue for SA immigrant women (Kernohan 1996, Sadler et al. 2001, Szczepura et al. 2008) or both genders (Lobb et al. 2013, Gesink et al. 2014). In addition, a loss of social support upon immigration was another barrier (Szczepura et al. 2003, Ahmad et al. 2004), which links to dependence on family for advice, support and language interpretation to access health and screening services.

A lack of time and financial concerns were common individual barriers for some SA immigrant women. These findings related to competing demands of family, children, work or household chores (Kernohan 1996, Sadler et al. 2001, Ahmad et al. 2004), as well as costs associated with taking unpaid time off work, securing childcare or transportation (Meana et al. 2001b, Lobb et al. 2013). Transportation barriers identified were challenges navigating the transit system, mobility issues and dependence on family for rides to screening (Meana et al. 2001b, Asanin \& Wilson 2007, Lee et al. 2010b, Ahmad et al. 2011).

Structural barriers. The structural barriers to cancer screening access included sociocultural barriers such as the gender or culture of family physician, and system barriers such as lack of a physician recommendation. Some SA immigrant women were fearful of having a male physician for a routine physical examination (Bottorff et al. 2001a, Oelke and Vollman 2007) or described difficulties communicating with a male physician (Karbani et al. 2011); while others stated that it was unacceptable to be examined by a male physician (Black \& Zsoldos 2003, Asanin \& Wilson 2007). The preference for a female healthcare provider with or without the same cultural background was a common attitude among SA immigrants (Bottorff et al. 2001a, Meana et al. 2001a, Black \& Zsoldos 2003, Pfeffer 2004, Thomas et al. 2005, Asanin \& Wilson 2007, Oelke and Vollman 2007, Lee et al. 2010b). A lack of female primary care providers as well as organisational structures to facilitate access, such as personal reminders, were also reported barriers to cancer screening tests (Lobb et al. 2013).

Health education programmes that are not culturally sensitive, used inconsistent messages and lacked attention to appropriate level of health literacy and credible dissemination channels (i.e. places of worship) were also cited barriers to cancer screening (Lobb et al. 2013). While having a physician from the same culture or language was a preference for some SA immigrant women and men (Bottorff et al. 1998, Oelke \& Vollman 2007, Lee et al. 2010b, Lobb et al. 
2013), and may facilitate screening, a physician from the same culture sometimes had the opposite effect because physicians did not recommend screening out of respect for modesty and comfort (Bottorff et al. 2001a, Somanchi et al. 2010).

The lack of a doctor recommendation for screening was a common barrier identified among SA immigrant females (Rudat 1994, Bottorff et al. 2001a, Somanchi et al. 2010). Having a physician recommendation was associated with an increased likelihood of having breast or cervical screening (Gupta et al. 2002, Boxwala et al. 2010, Somanchi et al. 2010). Other barriers included a lack of local access to screening services (Thomas et al. 2005, Asanin \& Wilson 2007) and a lack of health insurance among SA immigrants residing in the US (De Alba et al. 2005, Wong et al. 2005, Islam et al. 2006, Gomez et al. 2007, Glenn et al. 2009, Lee et al. 2010b, Pourat et al. 2010, Somanchi et al. 2010, Misra et al. 2011, Mehrotra et al. 2012).

\section{Theme 4: gender differences}

Gender influenced screening uptake. SA immigrant women in the UK, US and Canada reported lower screening rates for breast or cervical cancer screening (Kernohan 1996, Rashidi \& Rajaram 2000, Gupta et al. 2002, Ahmad \& Stewart 2004, Islam et al. 2006, Boxwala et al. 2010, Mehrotra et al. 2012, Menon et al. 2012, Hasnain et al. 2014), and these were also low when compared with non-Asian-born populations (Rudat 1994, Sutton et al. 2001, Chaudhry et al. 2003, Webb et al. 2004, Quan et al. 2006, Kagawa-Singer et al. 2007, McDonald \& Kennedy 2007, Woltman \& Newbold 2007, Brotto et al. 2008, Amankwah et al. 2009, Lofters et al. 2010, Bansal et al. 2012). In some studies, specific screening rates were lower among SA immigrants compared to other ethno-cultural populations in the US (De Alba et al. 2005, Gomez et al. 2007, Lee et al. 2010a, Pourat et al. 2010, Bansal et al. 2012, Patel et al. 2012) and Canada (Brotto et al. 2008, Amankwah et al. 2009, Lofters et al. 2010).

For colorectal cancer screening, SA immigrant men in the US had higher screening rates or odds of screening compared with SA immigrant women (Glenn et al. 2009, Misra et al. 2011). Low rates of breast and colorectal cancer screening were common among SA immigrants overall in the UK; however, Muslim SA immigrant women had the lowest rates compared to SA immigrant men and non-Asian British-born women (Szczepura et al. 2008, Price et al. 2010).

Factors associated with cancer screening included the length of residence in the new host country, having a usual source of care and level of education attainment. SA immigrant women settled for more years in the new host country had greater uptake of breast or cervical cancer screening (Choudhry et al. 1998, Meana et al. 2001b, Chaudhry et al. 2003, Ahmad \& Stewart 2004, McDonald \& Kennedy 2007, Woltman \& Newbold 2007, Amankwah et al. 2009, Glenn et al. 2009, Wu \& Ronis 2009, Boxwala et al. 2010, Lofters et al. 2010, Pourat et al. 2010, Somanchi et al. 2010, Surood \& Lai 2010, Misra et al. 2011, Menon et al. 2012). Greater acculturation or language acculturation within the host country was associated with information seeking behaviours, mammography or FOBT uptake and clinical breast examination among SA immigrants (Marfani et al. 2013, Menon et al. 2014). In one US study, SA immigrants eligible for breast, cervical or colorectal cancer screening were up to date with screening $(50 \%-79 \%)$; the sample was comprised of two-thirds male, had higher education and insurance coverage, and were English speaking (Bharmal \& Chaudhry 2012).

A usual source of healthcare provision was also associated with increased cancer screening rates among SA immigrants (Chaudhry et al. 2003, Islam et al. 2006, De Alba et al. 2005, Kagawa-Singer et al. 2007, Amankwah et al. 2009, Lofters et al. 2010, Pourat et al. 2010, Bharmal \& Chaudhry 2012), as was higher income and higher education level (Meana et al. 2001b, Gupta et al. 2002, Chaudhry et al. 2003, Islam et al. 2006, Amankwah et al. 2009, Glenn et al. 2009, Misra et al. 2011). In contrast, more recent SA immigrant men and women tend to have lower cancer screening rates (Wong et al. 2005, McDonald \& Kennedy 2007, Wu \& Ronis 2009, Boxwala et al. 2010).

\section{Discussion}

To our knowledge, this scoping study is the first to provide a comprehensive review of cancer screening for breast, cervical and colorectal cancers among SA immigrants in the UK, US and Canada. Four overarching themes emerged from the thematic analysis: (i) beliefs and attitudes towards cancer and screening; (ii) lack of knowledge of cancer and screening; (iii) barriers to access; and (iv) gender differences in screening uptake and their associated factors. The findings can inform practice and research aimed at reducing identified disparities in cancer screening. While approximately two-thirds of studies focused on female-specific cancers with SA immigrant women, the findings provide valuable insights into factors that influence screening decisions among women and men taking into consideration the heterogeneity of SA immigrant populations.

Two reviews (Elkan et al. 2006, Sokal 2010) examined cancer screening among SA immigrants; however, both included only studies undertaken in the 
UK. Sokal's (2010) study focused primarily on breast and cervical cancer screening literature among SA immigrant women residing in Britain. While several key findings were similar to themes identified in this review such as low self-perceived risk, language barrier and preference for female healthcare provider, Sokal (2010) included a small pool of studies, did not address colorectal cancer screening and lacked a gender perspective. The other critical review (Elkan et al. 2006) examined the UK literature undertaken with ethnic minority groups and the use of cancer services including screening, and had some findings similar to our study such as fear and fatalism, and lack of knowledge of screening. However, this study was limited due to its broad focus on cancer services, lack of data on barriers and facilitators to screening, and exclusion of colorectal cancer screening (Elkan et al. 2006). Finally, an integrative review (Wu et al. 2005) examined factors that influenced uptake of breast cancer screening among four Asian immigrant groups living in the US. However, this review was limited in reporting on Asian Indian women's perspectives from the US as well as sociocultural factors related to screening.

Evidence from dominant themes identified in this study can be used in the development of culturally sensitive interventions to promote cancer screening in SA immigrant communities. First, the collectivist nature of the SA culture was a dominant sub-theme from studies in the UK, US and Canada. Thus, consideration of family in the development of community-based strategies that enhance knowledge and awareness of cancer and population-based screening programmes to promote increased uptake may be of benefit. Including family may help address barriers associated with low English language proficiency and facilitate encouragement, accompaniment or provision of transportation.

Second, the reviewed literature provided insights into how health promotion messages may be framed within a more holistic manner. Although this subtheme was identified only in North American studies, it provides an initial conceptualisation for developing health messages to be inclusive of SA immigrants' cultural beliefs. Health promotion focused on primary prevention strategies such as healthy diet and physical activity is integral to the prevention of cancer (World Cancer Research Fund [WCRF]/AICR 2009). Messages to enhance knowledge and awareness that encompass both instructions on the screening test to be performed and the knowledge surrounding evidence-based primary prevention messaging associated with the specific type of cancer and risk factors may be more effective. Promoting screening to SA immigrants will also require respect and acknowledgement of traditional cultural values, beliefs and views of health.

The primary care provider should be integral in promoting messages through formalised healthcare access via the routine health examination. Consistency and alignment of health promotion messages and efforts of public health and primary care could lead to increased uptake of cancer screening. Public health and primary care collaboration, a current focus of attention in reducing health disparities among ethnic and minority populations (Institute of Medicine, 2012), has the potential to improve relationships and satisfaction with family physician care.

Third, lack of knowledge of breast, cervical or colorectal cancers and the impact of early detection on health outcomes was a dominant theme across all countries, highlighting the need to incorporate this information when creating culturally sensitive messaging. This information might also address identified fatalistic beliefs. In addition, promoting access to cancer screening tests will require an understanding of how to access the healthcare system for preventive health examinations.

Fourth, raising awareness that the risk of common cancers become similar to those of the host country of settlement over time might also increase uptake of screening services (Hislop et al. 2007). A self-perceived risk of cancer often reflects knowledge of the prevalence of common diseases in one's native country. This would also clarify that screening takes place in the absence of symptoms and could also mitigate the perception that screening is not needed if one is healthy. That is, cancer screening could be promoted as a method to maintain health.

Finally, the planning of health promotion and awareness strategies to increase uptake of cancer screening should include members of local SA immigrants in the community of interest in the development of locally driven initiatives to address their unique belief systems and needs. Reducing inequities in screening involves consideration and modifications to current health education programming and the improvement of culturally appropriate messaging to address health literacy and access (Lobb et al. 2013). The use of community-based media dissemination through ethnic papers or TV in SA immigrants' language was a method to overcome language barriers among SA immigrants in several reviewed studies (Ahmad et al. 2004, Austin et al. 2009). Discussion circles or educational seminars held in community settings such as recreation centres or temples were recommendations proposed by SA immigrants as a means to increase uptake in a number of included 
studies (Ahmad et al. 2004, Austin et al. 2009). In addition, the primary care physician was viewed as important to facilitating access to information on healthy lifestyle behaviours, prevention and recommending cancer screening through the use of referral letters or in-person dialogues (Ahmad et al. 2004, Austin et al. 2009, Lobb et al. 2013). Translation and interpretation services are a strategy to facilitate discussions among physicians and their SA immigrant clients.

This study underscores the cultural and settlement heterogeneity of SA immigrants including their unique historical, religious and political histories, migration patterns, socioeconomic status and contextual differences among countries of settlement. For example, the structural barriers of lack of local access and a physician recommendation were specific to the US and Canada. The structural organisation of where healthcare services are situated in North America results in access barriers including transportation issues. In North America, there is also reliance on primary care physicians to promote population-based screening for breast, cervical and colorectal cancers. In contrast, the UK's population-based screening programmes have been promoted more widely, and have been in existence for longer incorporating quality indicators and focused attention on recruitment strategies at all levels including primary care. Moreover, lack of insurance coverage was specific to the US only. Consequently, to increase cancer screening, context-specific strategies will be required based on available cancer prevention programmes, health services organisation and access in each country, along with specific community characteristics.

The initial intent of this study was to examine factors that influenced uptake and access to colorectal cancer screening among SA immigrants; however, due to low citations returned, the research question was broadened to include studies on access to all population-based cancer screening. Nevertheless, this study identified a scholarly gap around colorectal cancer screening among SA immigrants. Thus, it is unclear whether factors that influence uptake in SA immigrant women for female cancer screening are similar for colorectal cancer screening. Future research may include an examination of colorectal cancer screening behaviours among SA immigrants including barriers and facilitators to all colorectal cancer screening modalities. The unique difference between colorectal cancer screening tests includes the ability to understand directions for completing a home test using the FOBT kit or home preparation for a flexible sigmoidoscopy or colonoscopy (Rakowski \& Breslau 2004). Gender differences in colorectal cancer screening preferences among SA immigrants also present an opportunity for further research.

Additional research may also explore if the social influence of family mediates intention or uptake of cancer screening in SA immigrants. While the preference for holistic approach to health was specific to North American studies among mostly female SA immigrants, future research could examine if this belief is representative of SA immigrant men and women in other settings. Furthermore, gender-specific factors associated with screening uptake could be expanded upon to elucidate further gender differences, so that promotion efforts may be gender sensitive.

A number of limitations are noted. Despite a structured and comprehensive strategy to identify included studies and reports based on inclusion/ exclusion criteria, some relevant studies may have been missed. In the process of summarising key themes, the subjective position of the primary reviewer of full-text articles may have inadvertently introduced bias in decision-making. However, a team-based approach contributed to the study selection determination throughout each step, and a consistent approach was used to chart findings of included studies. Furthermore, Arksey and $\mathrm{O}^{\prime} \mathrm{Mal}-$ ley's (2005) framework made the process transparent minimising this bias. The included studies/reports in this scoping study encompassed a diverse sample of SA immigrants, and in some studies, distinct subgroups were not always identified. Therefore, it is important to take into consideration the heterogeneity of the SA immigrant populations residing in the UK, US and Canada as they hold multiple belief systems, different historical and political histories, varying socioeconomic status and unique environmental contexts of the country of settlement.

\section{Conclusion}

This scoping study makes a new contribution by synthesising and thematically analysing current knowledge about factors influencing cancer screening decisions among SA immigrants. The findings have illuminated important sociocultural beliefs of family, cancer, self-perceived risks of common cancers in host countries, holistic approaches to health and attitudes towards the sensitive nature of engaging in screening procedures. Identified barriers and associated factors were also of relevance to gain a perspective on ways to minimise barriers and improve screening uptake among SA immigrants. Locally driven strategies that incorporate SA immigrants' sociocultural beliefs and 
values of family and a holistic approach to health both have the potential to increase screening for breast, cervical and colorectal cancers among this heterogeneous population. In addition, an examination of the specific needs of a SA immigrant community will be necessary so that promotion strategies are geared towards minimising specific local barriers to access and screening. While the literature on cancer screening in this population is growing, there is limited evidence about colorectal cancer screening and gender-specific factors that influence cancer screening uptake. Further research can address this gap and enhance the current body of literature on health inequities.

\section{References}

Ahmad F. \& Stewart D.E. (2004) Predictors of clinical breast examination among South Asian immigrant women. Journal of Immigrant Health 6 (3), 119-126.

Ahmad F., Shik A., Vanza R., Cheung A., George U. \& Stewart D.E. (2004) Population health promotion strategies among Chinese and East Indian immigrant women. Women and Health 40 (1), 21-40.

Ahmad F., Cameron J.I. \& Stewart D.E. (2005) A tailored intervention to promote breast cancer screening among SA immigrant women. Social Science and Medicine 60 (3), $575-586$

Ahmad F., Mahmood S., Pietkiewicz I., McDonald L. \& Ginsburg O. (2011) Concept mapping with South Asian immigrant women: barriers to mammography and solutions. Journal of Immigrant and Minority Health 14 (2), 242-250.

Alexandraki I. \& Mooradian A.D. (2010) Barriers related to mammography use for breast cancer screening among minority women. Journal of the National Medical Association 102 (3), 206-218.

Amankwah E., Ngwakongnwi E. \& Quan H. (2009) Why many visible minority women in Canada do not participate in cervical cancer screening. Ethnicity and Health $\mathbf{1 4}$ (4), 337-349.

Arksey H. \& O'Malley L. (2005) Scoping studies: towards a methodological framework. International Journal of Social Research Methodology 8 (1), 19-31.

Asanin J. \& Wilson K. (2007) I spent nine years looking for doctor: exploring access to health care among immigrants in Mississauga, Ontario, Canada. Social Science and Medicine 66, 1271-1283.

Austin K.L., Power E., Solarin I., Atkin W.S., Wardle J. \& Robb K.A. (2009) Perceived barriers to flexible sigmoidoscopy screening for colorectal cancer among UK ethnic minority groups: a qualitative study. Journal of Medical Screening 16 (4), 174-179.

Ballard R. (2003) The South Asian presence in Britain and its transnational connections. In: H. Singh \& S. Vertovec (Eds) Culture and Economy in the Indian Diaspora, pp. 197222. Routledge, London.

Banning M. \& Hafeez H. (2010) A two-centre study of Muslim women's views of breast cancer and breast health practices in Pakistan and the UK. Journal of Cancer Education 25 (3), 349-353.
Bansal N., Bhopal R.S., Steiner M.F.C. \& Brewster D.H. (2012) Major ethnic group differences in breast cancer screening uptake in Scotland are not extinguished by adjustment for indices of geographical residence, area deprivation, long term illness and education. British Journal of Cancer 106, 1361-1366.

Baron R.C., Rimer R.K., Coates R.J. et al. (2008) Clientdirected interventions to increase access to breast, cervical, and colorectal cancer screening: a systematic review. American Journal of Preventive Medicine 35 (1 Suppl.), S56-S66.

Bharmal N. \& Chaudhry S. (2012) Preventive health services delivery to South Asians in the United States. Journal of Immigrant Minority Health 14, 797-802.

Bierman A.S., Angus J., Ahmad F. et al. (2009/2010) Access to health care services. In: A.S. Bierman (Ed) Project for an Ontario Women's Health Evidence Report: Volume 1. Toronto, Ontario. Available at: http://www.powerstudy.ca/thepower-report (accessed on 12/6/2012).

Black M. \& Zsoldos J. (2003) Lay Health Educators to Enhance Cancer Screening Summary Report of Focus Groups: Planning with Women from Four Communities. Hamilton Public Health and Community Services, Hamilton, Ontario (Unpublished).

Bottorff J.L., Johnson J.L., Bhagat R., Grewal S., Balneaves L., Clarke H. \& Hilton B.A. (1998) Beliefs related to breast health practices: the perceptions of South Asian women living in Canada. Social Science and Medicine 47 (12), 2075-2085.

Bottorff J.L., Balneaves L.G., Sent L., Grewal S. \& Browne A.J. (2001a) Cervical cancer screening in ethno-cultural groups: case studies in women-centered care. Women and Health 33 (3), 29-46.

Bottorff J.L., Johnson J.L., Venables L.J., Grewal S. \& Popa N. (2001b) Voices of immigrant South Asian women: expressions of health concerns. Journal of Health Care for the Poor and Underserved 12 (4), 392-403.

Boxwala F.L., Bridgemohan A., Griffith D.M. \& Soliman A.S. (2010) Factors associated with breast cancer screening in Asian Indian women in metro-Detroit. Journal of Immigrant Minority Health 12, 534-543.

Brotto L.A., Chou A.Y., Singh T. \& Woo J.S. (2008) Reproductive health practices Among Indian, Indo-Canadian, Canadian East Asian, and Euro-Canadian women: the role of acculturation. Journal of Obstetrics and Gynaecology in Canada 30 (3), 229-238.

Chaudhry S., Fink A., Gelberg L. \& Brook R. (2003) Utilization of Papanicolaou smears by South Asian women living in the United States. Journal of General Internal Medicine 18, 377-384.

Choudhry U.K. (1998) Health promotion among immigrant women from India living in Canada. Journal of Nursing Scholarship 30 (3), 269-274.

Choudhry U.K., Srivastava R. \& Fitch M.I. (1998) Breast cancer detection practices of South Asian women: knowledge, attitudes, and beliefs. Oncology Nursing Forum 25, 1693-1701.

De Alba I., Ngo-Metzger Q., Sweningson J.M. \& Hubbell F.A. (2005) Pap smear use in California: are we closing the racial/ethnic gap? Preventive Medicine 40 (6), 747-755.

Elkan R., Avis M., Cox K. et al. (2006) The reported views and experiences of cancer service users from minority ethnic groups: a critical review of the literature. European Journal of Cancer Care 16, 109-121. 
Forbes L.J.L., Atkins L., Thurnham A., Layburn J., Haste F. \& Ramirez A.J. (2011) Breast cancer awareness and barriers to symptomatic presentation among women from different ethnic groups in East London. British Journal of Cancer 105, 1474-1479.

Gesink D., Mihic A., Antal J. et al. (2014) Who are the under- and never-screened for cancer in Ontario: a qualitative investigation. BMC Public Health 14, 495.

Glenn B.A., Chawla N., Surani Z. \& Bastani R. (2009) Rates and socio-demographic correlates of cancer screening among SAs. Journal of Community Health 34 (2), 113-121.

Gomez S.L., Tan S., Keegan T.H. \& Clarke C.A. (2007) Disparities in mammographic screening for Asian women in California: a cross-sectional analysis to identify meaningful groups for targeted intervention. BMC Cancer 7, 201.

Gupta A., Kumar A. \& Stewart D.E. (2002) Cervical cancer screening among South Asian women in Canada: the role of education and acculturation. Health Care Women International 23 (2), 123-134.

Hanson K., Montgomery P., Bakker D. \& Conlon M. (2009) Factors influencing mammography participation in Canada: an integrative review of the literature. Current Oncology 16 (3), 65-75.

Hasnain M., Menon U., Ferrans C.E. \& Szalacha L. (2014) Breast cancer screening practices among first-generation immigrant Muslim women. Journal of Women's Health 23 (7), 602-612.

Hislop T.G., Bajdick C.D., Saroa S.R., Yeole B.B. \& Barroetavena M.C. (2007) Cancer incidence in Indians from three areas: Delhi and Mumbai, India, and British Columbia, Canada. Journal of Immigrant and Minority Health 9, 221-227.

Hossain A., Sehbai A., Abrahan R. \& Abraham J. (2008) Cancer health disparities among Indian and Pakistani immigrants in the United States: a surveillance, epidemiology, and end results-based study from 1988-2003. Cancer 113 (6), 1423-1430.

Institute of Medicine (2012) Primary Care and Public Health: Exploring Integration to Improve Population Health, pp. 94 101. National Academies Press, Washington, DC. Available at: http:// www.iom.edu/Reports/2012/Primary-Care-andPublic-Health.aspx

Islam N., Kwon S.C., Senie R. \& Kathuria N. (2006) Breast and cervical cancer screening among South Asian women in New York City. Journal of Immigrant and Minority Health 8 (3), 211-221.

Jain R.V., Mills P.K. \& Parikh-Patel A. (2005) Cancer incidence in the SA population in California. Journal of Carcinogenesis 4, 21-33.

Johnson C.E., Mues K.E., Mayne S.L. \& Kiblawi A.N. (2008) Cervical cancer screening among immigrants and ethnic minorities: a systematic review using the Health Belief Model. Journal of Lower Genital Tract Disease 12 (3), 232-241.

Kagawa-Singer M., Pourat N., Breen N., Coughlin S., Mclean T.A. \& McNeel T.S. (2007) Breast and cervical cancer screening rates of subgroups of Asian American women. Medical Care Research and Review 64 (6), 706-730.

Karbani G., Lim J.N.W., Hewison J., Atkin K., Horgan K., Lansdown M. \& Chu C.E. (2011) Culture, attitude and knowledge about breast cancer and preventive measures: a qualitative study of South Asian breast cancer patients in the UK. Asian Pacific Journal of Cancer Prevention 12, 1619-1626.
Kernohan E.E.M. (1996) Evaluation of a pilot study for breast and cervical cancer screening with Bradford's minority ethnic women: a community development approach, 1991-93. British Journal of Cancer 74 (Suppl), S42-S46.

Lee H.Y., Ju E., Vang P.D. \& Lundquist M. (2010a) Breast and cervical cancer screening among Asian American women and Latinas: does race/ethnicity matter? Journal of Women's Health 19 (10), 1877-1884.

Lee S., Martinez G., Ma G.X., Hsu C.E., Robinson E.S., Bawa J. \& Juon H.-S. (2010b) Barriers to health care access in 13 Asian American communities. American Journal of Health Behavior 34 (1), 21-30.

Lee H.Y., Lundquist M., Ju E., Luo X. \& Townsend A. (2011) Colorectal cancer screening disparities in Asian Americans and Pacific Islanders: which groups are most vulnerable. Ethnicity and Health 16 (6), 501-518.

Levac D., Colquhoun H. \& O’Brien K.K. (2010) Scoping studies: advancing the methodology. Implementation Science 5, 69.

Lobb R., Pinto A.D. \& Lofters A. (2013) Using concept mapping in the knowledge-to-action process to compare stakeholder opinions on barriers to use of cancer screening among South Asians. Implementation Science 8, 37.

Lofters A.K., Hwang S.W., Moineddin R. \& Glazier R.H. (2010) Cervical cancer screening among urban immigrants by region of origin: a population-based cohort study. Preventive Medicine 51 (6), 509-516.

Marfani F., Rimal R.N. \& Hee-Soon J. (2013) Understanding immigrant women's information needs: role of acculturation in breast cancer prevention among immigrant Asian Indian women. Journal of Applied Communication Research 41 (2), 126-140.

Matin M. \& LeBaron S. (2004) Attitudes toward cervical cancer screening among Muslim women: a pilot study. Women and Health 39 (3), 63-77.

Mays N., Pope C. \& Popay J. (2005) Systematically reviewing qualitative and quantitative evidence to inform management and policy-making in the health field. Journal of Health Services Research and Policy 10 (Suppl 1), S6-S20.

McDonald J.T. \& Kennedy S. (2007) Cervical cancer screening and minority women in Canada. Journal of Immigrant Minority Health 9, 323-334.

Meana M., Bunston T., George U., Wells L. \& Rosser W. (2001a) Older immigrant Tamil women and their doctors: attitudes toward breast cancer screening. Journal of Immigrant Health 3 (1), 5-13.

Meana M., Bunston T., George U., Wells L. \& Rosser W. (2001b) Influences on breast cancer screening behaviors in Tamil immigrant women 50 years old and over. Ethnicity and Health 6 (3-4), 179-188.

Mehrotra N., Gaur S. \& Petrova A. (2012) Health care practices of the foreign born Asian Indians in the United States: a community based survey. Journal of Community Health 37, 328-334.

Menon U., Szalacha L.A. \& Prabhughate A. (2012) Breast and cervical cancer screening among South Asian immigrants in the United States. Cancer Nursing 35 (4), 278-287.

Menon U., Szalacha L.A., Prabhughate A. \& Kue J. (2014) Correlates of colorectal cancer screening among South Asian immigrants in the United States. Cancer Nursing 37 (1), E19-E27. 
Misra R., Menon U., Vadaparampil S.T. \& Belue R. (2011) Age- and sex-specific cancer prevention and screening practices among Asian Indian immigrants in the United States. Journal of Investigative Medicine 59 (5), 787-792.

Oelke N.D. \& Vollman A.R. (2007) "Inside and outside": Sikh women's perspectives on cervical cancer screening. Canadian Journal of Nursing Research 39 (1), 174-189.

Patel V.V., Rajpathak S. \& Karasz A. (2012) Bangladeshi immigrants in New York City: a community based health needs assessment of a hard to reach population. Journal of Immigrant and Minority Health 14, 767-773.

Pfeffer N. (2004) Screening for breast cancer: candidacy and compliance. Social Science and Medicine 58, 151-160.

Poonawalla I.B., Goyal S., Mehrotra N., Allicock M. \& Balasubramanian B.A. (2014) Attitudes of South Asian women to breast health and breast cancer screening: findings from a community based sample in the United States. Asian Pacific Journal of Cancer Prevention 15 (20), 8719-8724.

Pourat N., Kagawa-Singer M., Breen N. \& Sripipatana A. (2010) Access versus acculturation: identifying modifiable factors to promote cancer screening among Asian American women. Medical Care 48 (12), 1088-1096.

Price C.L., Szczepura A.K., Gumber A.K. \& Patnick J. (2010) Comparison of breast and bowel cancer screening uptake patterns in a common cohort of South Asian women in England. BMC Health Services Research 10, 103.

Quan H., Fong A., De Coster C., Wang J., Musto R., Noseworthy T.W. \& Ghali W.A. (2006) Variation in health services utilization among ethnic populations. Canadian Medical Association Journal 174 (6), 787-791.

Rakowski W. \& Breslau E.S. (2004) Perspectives on behaviorual and social science research on cancer screening. Cancer 101 (5 Suppl), 1118-1130.

Randhawa G. \& Owens A. (2004) The meanings of cancer and perceptions of cancer services among South Asians in Luton, UK. British Journal of Cancer 91, 62-68.

Rashidi A. \& Rajaram S.S. (2000) Middle Eastern Asian Islamic women and breast self-examination needs assessment. Cancer Nursing 23 (1), 64-70.

Rastogi T., Devesa S., Mongtani P., Mathew A., Cooper N., Kao R. \& Sinha R. (2008) Cancer incidence rates among South Asians in four geographic regions: India, Singapore, UK and US. International Journal of Epidemiology 37, 147-160.

Robb K.A., Solarin I., Power E., Atkin W. \& Wardle J. (2008) Attitudes to colorectal cancer screening among ethnic minority groups in UK. BMC Public Health 8, 34.

Robb K., Wardle J., Stubbings S. et al. (2010) Ethnic disparities in knowledge of cancer screening programmes in the UK. Journal of Medical Screening 17 (3), 125-131.

Rudat K. (1994) Black and Minority Ethnic Groups in England: Health and Lifestyles. Health Education Authority, London. Available at: http://www.nice.org.uk/nicemedia/documents/black_minorities_england94.pdf (accessed on 14/ 6/2012).

Sadler G.R., Dhanjal S.K., Shah N.B., Shah R.B., Ko C., Anghel M. \& Harshburger R. (2001) Asian Indian women: knowledge, attitudes and behaviors toward breast cancer early detection. Public Health Nursing $\mathbf{1 8}$ (5), 357-363.

Smith L.K., Botha J.L., Benghiat A. \& Steward W.P. (2003) Latest trends in cancer incidence among UK South Asians in Leicester. British Journal of Cancer 89, 70-73.
Sokal R. (2010) A critical review of the literature on the uptake of cervical and breast screening in British South Asian women. Quality in Primary Care 18, 251-261.

Somanchi M., Juon H.S. \& Rimal R. (2010) Predictors of screening mammography among Asian Indian American women: a cross-sectional study in the Baltimore-Washington Metropolitan area. Journal of Women's Health 19 (3), 433-441.

Statistics Canada (2008) Canada's Ethno-cultural Mosaic 2006 Census: Provinces and Territories. Statistics Canada Catalogue no. 97-562-X, Ottawa, Ontario. Available at: http:// www12.statcan.ca/census-recensement/2006/as-sa/97-562/ pdf/97-562-XIE2006001.pdf (accessed on 3/2/2012).

Surood S. \& Lai D.W. (2010) Impact of culture on use of western health services by older SA Canadians. Canadian Journal of Public Health 101 (2), 176-180.

Sutton G.C., Storer A. \& Rowe K. (2001) Cancer screening coverage of South Asian women in Wakefield. Journal of Medical Screening 8 (4), 183-186.

Szczepura A., Johnson M., Orbell S., Gumber A., O'Sullivan I., Clay D. \& Owen D. (2003) Ethnicity: UK Colorectal Cancer Screening Pilot - Final Report. National Health Services Cancer screening programmes 2003. Available at: http:// www.cancerscreening.nhs.uk/bowel/pilot-evaluation.html (accessed on 3/2/2012).

Szczepura A., Price C. \& Gumber A. (2008) Breast and bowel cancer screening uptake patterns over 15 years for SA ethnic minority populations, corrected for differences in socio-demographic characteristics. BioMed Central Public Health 8, 346.

Taskila T., Wilson S., Damery S., Roalfe A., Redman V., Ismail T. \& Hobbs R. (2009) Factors affecting attitudes toward colorectal cancer screening in the primary care population. British Journal of Cancer 101, 250-255.

Thomas V.N., Saleem T. \& Abraham R. (2005) Barriers to effective uptake of cancer screening among black and minority ethnic groups. International Journal of Palliative Nursing 11 (11), 562-571.

Tran K., Kaddatz J. \& Allard P. (2005) South Asians in Canada: Unity through Diversity. Canadian Social Trends Statistics Canada Catalogue No. 11-008. Available at: http:/ / www5.statcan.gc.ca/bsolc/olc-cel/olc-cel?catno=11008-X20050028455\&lang=eng (accessed on 3/2/2012).

UK Census (2011) 2011 Census for England and Wales. Available at: http://www.statistics.gov.uk/census2001/census2001.asp (accessed on 3/2/2012).

United States Preventive Services Task Force (2008) Screening for colorectal cancer: U. S. Preventive Services Task Force recommendation statement. Annals of Internal Medicine 149 (9), 627-637.

United States Preventive Services Task Force (2009) Screening for breast cancer: US Preventive Services Task Force recommendation Statement. Annals of Internal Medicine 151 (10), 716-726.

United States Preventive Services Task Force (2012) Screening for cervical cancer: US Preventive Services Task Force recommendation Statement. Annals of Internal Medicine 156 (12), 880-891.

US Census Bureau (2010) Overview of race and Hispanic origin. Available at: http://www.census.gov/prod/cen2010/ briefs/c2010br-02.pdf (accessed on 3/2/2012).

Virk R., Gill S., Yoshida E., Radley S. \& Salh B. (2010) Racial differences in the incidence of colorectal cancer. Canadian Journal of Gastroenterology 24 (1), 47-51. 
Webb R., Richardson J., Esmail A. \& Pickles A. (2004) Uptake of cervical screening by ethnicity and place of birth: a population-based cross-sectional study. Journal of Public Health 26 (3), 293-296.

Woltman K.J. \& Newbold K.B. (2007) Immigrant women and cervical cancer screening uptake: a multilevel analysis. Canadian Journal of Public Health 98 (6), 470-475.

Wong S.T., Gildengorin G., Nguyen T. \& Mock J. (2005) Disparities in colorectal cancer screening rates among Asian Americans and Non-Latino Whites. Cancer 104 (12 Suppl), 2940-2947.

World Cancer Research Fund [WCRF]/AICR (2009) Food, nutrition, physical activity and the prevention of cancer: a global perspective online: recommendations. Available at: http:// www.dietandcancerreport.org/?p=recommendations (accessed on $9 / 8 / 2014)$.

Wu T.Y. \& Ronis D. (2009) Correlates of recent and regular mammography screening among Asian-American women. Journal of Advanced Nursing 65 (11), 2434-2446.

Wu T.Y., Bancroft J. \& Guthrie B. (2005) An integrative review on breast cancer screening practice and correlates among Chinese, Korean, Filipino, and Asian Indian American women. Health Care for Women International 26, 225-246.

Wu T.Y., West B., Chen Y.W. \& Hergert C. (2006) Health beliefs and practices related to breast cancer screening in Filipino, Chinese and Asian-Indian women. Cancer Detection and Prevention 30 (1), 58-66. 